\title{
Study of the Analytical Treatment of the (2+1)-Dimensional Zoomeron, the Duffing and the SRLW Equations via a New Analytical Approach
}

\author{
Jalil Manafian $^{1} \cdot$ Mehrdad Lakestani $^{1} \cdot$ Ahmet Bekir $^{2}$
}

\begin{abstract}
In this paper, we applied the improved tan $(\Phi(\xi) / 2)$-expansion scheme for the $(2+1)$-dimensional Zoomeron, the Duffing and the symmetric regularized long wave equations and exact particular solutions have been found. The exact particular solutions containing four types hyperbolic function solution, trigonometric function solution, exponential solution and rational solution. We obtained the further solutions comparing with other methods as sine-cosine function method (Qawasmeh in J Math Comput Sci 3:1475-1480, 2013). Recently this method is developed for searching exact travelling wave solutions of nonlinear partial differential equations. It is shown that this method, with the help of symbolic computation, provide a straightforward and powerful mathematical tool for solving nonlinear partial differential equations.
\end{abstract}

Keywords Improved tan $(\Phi(\xi) / 2)$-expansion method - The (2+1)-dimensional Zoomeron, the Duffing and the symmetric regularized long wave (SRLW) equations - Traveling wave

\section{Introduction}

During the last decades, some major contributions have been made to both the theory and applications of the nonlinear partial differential equations motivated by various practical engineering and physical problems. These applications cross diverse disciplines, such as

Ahmet Bekir

abekir@ogu.edu.tr

Jalil Manafian

j_manafianheris@tabrizu.ac.ir

Mehrdad Lakestani

lakestani@tabrizu.ac.ir

1 Department of Applied Mathematics, Faculty of Mathematical Science, University of Tabriz, Tabriz, Iran

2 Department of Mathematics - Computer, Eskişehir Osmangazi University, 26480 Eskisehir, Turkey 
chemical physics [1], viscoelasticity [2], electricity [3], biomedical engineering [1], fluid mechanics [4] and other sciences. In fact, it has been found that many models in mathematics and physics are described by nonlinear partial differential equations (NPDEs). The theory of solitons, the most important side in applications to NPDEs, has contributed to understanding many experiments in mathematical physics. Thus, it is of interest to evaluate new solutions of these equations. In the present paper, based on the improved $\tan (\Phi(\xi) / 2)$-expansion method, we will consider an important equation, which is the $(2+1)$-dimensional Zoomeron $[5,6]$ with the form

$$
\left(\frac{\mathrm{u}_{\mathrm{xy}}}{\mathrm{u}}\right)_{\mathrm{tt}}-\left(\frac{\mathrm{u}_{\mathrm{xy}}}{\mathrm{u}}\right)_{\mathrm{xx}}+2\left(\mathrm{u}^{2}\right)_{\mathrm{xt}}=0,
$$

where $\mathrm{u}=\mathrm{u}(\mathrm{x}, \mathrm{y}, \mathrm{t})$ is the unknown real function and the amplitude of the relevant wave mode. Equation (1) appears in a wide variety of physical. The Zoomeron equation was introduced by Calogero and Degasperis in 1976 [7]. The solitary wave solutions of the Zoomeron equation obtained by Abazari [8]. In [6] the reliable treatment of the $(2+1)$ dimensional Zoomeron equation have been surveyed by Alquran and Al-Khaled. Qawasmeh [5] have applied the sine-cosine function method to construct the traveling wave solutions for the (2+1)-dimensional Zoomeron equation, the Duffing equation and the SRLW equation. In [9], Khan and Akbar investigated solutions of the $(2+1)$-dimensional Zoomeron equation and the $(2+1)$-dimensional Burgers equation by using the MSE method and the Exp-function method. Also Bekir et al. [10] used the first integral method for constructing exact solutions of the Zoomeron and Klein-Gordon-Zakharov equations. As a second example we consider the Duffing equations as follows

$$
\mathrm{u}_{\mathrm{tt}}+\alpha \mathrm{u}+\beta \mathrm{u}^{3}=0
$$

this equation have solved by sine-cosine method [5]. Both integral and non-integral forcing terms for Duffing equation was solved by Balaji [11]. As a last example we consider the SRLW equation [5] as follows

$$
\mathrm{u}_{\mathrm{tt}}+\mathrm{u}_{\mathrm{xx}}+\mathrm{u}_{\mathrm{xxtt}}+\left(\mathrm{uu}_{\mathrm{x}}\right)_{\mathrm{t}}=0 .
$$

Authors of [12] was established exact travelling wave solutions of the symmetric regularized long wave (SRLW) by using analytical methods. Xu [13] applied of Exp-function method to SRLW equation. Cahnd and Malik [14] have found the exact solutions of some nonlinear evolution equations by using $\left(\mathrm{G}^{\prime} / \mathrm{G}\right)$-expansion method. Recently, a variety of powerful methods for seeking the explicit and exact solutions of nonlinear evolution equations have been proposed and developed. Among them are the Hirota's bilinear method [15], homotopy analysis method [16], variational iteration method [17], homotopy perturbation method [18,19], homogenous balance method [20], sine-cosine method [21], tanh-coth method [22], Bäcklund transformation [23], $\left(\frac{G^{\prime}}{G}\right)$-expansion method [24-27], Exp-function method [28-30], modified simple equation method [31-33], first integral method [34-37], functional variable method [38,39], and so on [40]. Here, we use of an effective method for constructing a range of exact solutions for the following NPDEs that in this article we developed solutions as well. The standard tanh method is well-known analytical method which first presented by Malfliet's [41] and developed in [41,42]. In [22], we applied the generalized tanh-coth method in for solving some NPDEs. Also in [43], the new approach of generalized $\left(G^{\prime} / G\right)$-expansion method to obtain exact traveling wave solutions of NLEEs is presented. In this paper we explain methods which are called the generalized tanh-coth and generalized $\left(G^{\prime} / G\right)$-expansion methods are presented to look for travelling wave solutions of nonlinear evolution equations. Authors of [44], obtained exact solutions for the integrable sixth-order 
Drinfeld-Sokolov-Satsuma-Hirota system by the generalized tanh-coth and generalized (G'/G)-expansion methods. Chand and Malik [14] have applied the (G'/G)-expansion method for finding the exact solutions of some nonlinear evolution equations. For further information in about these methods refer to Ref. [45-49]. The purpose of this paper is to obtain exact solutions of the (2+1)-dimensional Zoomeron, the Duffing and the SRLW equations and to determine the accuracy of the improved $\tan (\Phi(\xi) / 2)$-expansion method in solving these kind of problems. The paper is organized as follows: In "Description of improved $\tan (\Phi(\xi) / 2)$ expansion technique" section, we describe the improved $\tan (\Phi(\xi) / 2)$-expansion method. In "Illustrative Examples" section, we examine the (2+1)-dimensional Zoomeron, the Duffing and the SRLW equations respectively. Also a conclusion is given in "Conclusion" section. Finally some references are given at the end of this paper.

\section{Description of Improved $\tan (\Phi(\xi) / 2)$-Expansion Technique}

Step 1. We suppose that the given nonlinear fractional partial differential equation for $\mathrm{u}(\mathrm{x}, \mathrm{t})$ to be in the form

$$
\mathcal{N}\left(\mathrm{u}, \mathrm{u}_{\mathrm{x}}, \mathrm{u}_{\mathrm{t}}, \mathrm{u}_{\mathrm{xx}}, \mathrm{u}_{\mathrm{tt}}, \ldots\right)=0
$$

which can be converted to an ODE

$$
\mathcal{Q}\left(u, u^{\prime},-\mu u^{\prime}, u^{\prime \prime 2} u^{\prime \prime}, \ldots\right)=0,
$$

the transformation $\xi=\mathrm{x}-\mu \mathrm{t}$, is wave variable. Also, $\mathrm{k}, \mathrm{m}$ and $\mathrm{n}$ are constants to be determined later.

Step 2. Suppose the traveling wave solution of Eq. (5) can be expressed as follows:

$$
\mathrm{u}(\xi)=\mathrm{S}(\Phi)=\sum_{\mathrm{k}=0}^{\mathrm{m}} \mathrm{A}_{\mathrm{k}}\left[\mathrm{p}+\tan \left(\frac{\Phi(\xi)}{2}\right)\right]^{\mathrm{k}}+\sum_{\mathrm{k}=1}^{\mathrm{m}} \mathrm{B}_{\mathrm{k}}\left[\mathrm{p}+\tan \left(\frac{\Phi(\xi)}{2}\right)\right]^{-\mathrm{k}}
$$

where $A_{k}(0 \leq k \leq m)$ and $B_{k}(1 \leq k \leq m)$ are constants to be determined, such that $\mathrm{A}_{\mathrm{m}} \neq 0, \mathrm{~B}_{\mathrm{m}} \neq 0$ and $\Phi=\Phi(\xi)$ satisfies the following ordinary differential equation:

$$
\Phi^{\prime}(\xi)=\mathrm{a} \sin (\Phi(\xi))+\mathrm{b} \cos (\Phi(\xi))+\mathrm{c} .
$$

We will consider the following special solutions of Eq. (7):

Family 1: When $a^{2}+b^{2}-c^{2}<0$ and $b-c \neq 0$,

$$
\Phi(\xi)=-2 \arctan \left[-\frac{\mathrm{a}}{\mathrm{b}-\mathrm{c}}+\frac{\sqrt{\mathrm{c}^{2}-\mathrm{b}^{2}-\mathrm{a}^{2}}}{\mathrm{~b}-\mathrm{c}} \tan \left(\frac{\sqrt{\mathrm{c}^{2}-\mathrm{b}^{2}-\mathrm{a}^{2}}}{2}(\xi+\mathrm{C})\right)\right] .
$$

Family 2: When $\mathrm{a}^{2}+\mathrm{b}^{2}-\mathrm{c}^{2}>0$ and $\mathrm{b}-\mathrm{c} \neq 0$,

$$
\Phi(\xi)=-2 \arctan \left[-\frac{\mathrm{a}}{\mathrm{b}-\mathrm{c}}-\frac{\sqrt{\mathrm{b}^{2}+\mathrm{a}^{2}-\mathrm{c}^{2}}}{\mathrm{~b}-\mathrm{c}} \tanh \left(\frac{\sqrt{\mathrm{b}^{2}+\mathrm{a}^{2}-\mathrm{c}^{2}}}{2}(\xi+\mathrm{C})\right)\right] .
$$


Family 3: When $\mathrm{a}^{2}+\mathrm{b}^{2}-\mathrm{c}^{2}>0, \mathrm{~b} \neq 0$ and $\mathrm{c}=0$,

$$
\Phi(\xi)=2 \arctan \left[\frac{\mathrm{a}}{\mathrm{b}}+\frac{\sqrt{\mathrm{b}^{2}+\mathrm{a}^{2}}}{\mathrm{~b}} \tanh \left(\frac{\sqrt{\mathrm{b}^{2}+\mathrm{a}^{2}}}{2}(\xi+\mathrm{C})\right)\right] .
$$

Family 4: When $\mathrm{a}^{2}+\mathrm{b}^{2}-\mathrm{c}^{2}<0, \mathrm{c} \neq 0$ and $\mathrm{b}=0$,

$$
\Phi(\xi)=2 \arctan \left[-\frac{\mathrm{a}}{\mathrm{c}}+\frac{\sqrt{\mathrm{c}^{2}-\mathrm{a}^{2}}}{\mathrm{c}} \tan \left(\frac{\sqrt{\mathrm{c}^{2}-\mathrm{a}^{2}}}{2}(\xi+\mathrm{C})\right)\right] .
$$

Family 5: When $\mathrm{a}^{2}+\mathrm{b}^{2}-\mathrm{c}^{2}>0, \mathrm{~b}-\mathrm{c} \neq 0$ and $\mathrm{a}=0$,

$$
\Phi(\xi)=2 \arctan \left[\sqrt{\frac{\mathrm{b}+\mathrm{c}}{\mathrm{b}-\mathrm{c}}} \tanh \left(\frac{\sqrt{\mathrm{b}^{2}-\mathrm{c}^{2}}}{2}(\xi+\mathrm{C})\right)\right] .
$$

Family 6: When $\mathrm{a}=0$ and $\mathrm{c}=0$,

$$
\Phi(\xi)=\arctan \left[\frac{\mathrm{e}^{2 \mathrm{~b}(\xi+\mathrm{C})}-1}{\mathrm{e}^{2 \mathrm{~b}(\xi+\mathrm{C})}+1}, \frac{2 \mathrm{e}^{\mathrm{b}(\xi+\mathrm{C})}}{\mathrm{e}^{2 \mathrm{~b}(\xi+\mathrm{C})}+1}\right] .
$$

Family 7: When $b=0$ and $c=0$,

$$
\Phi(\xi)=\arctan \left[\frac{2 \mathrm{e}^{\mathrm{a}(\xi+\mathrm{C})}}{\mathrm{e}^{2 \mathrm{a}(\xi+\mathrm{C})}+1}, \frac{\mathrm{e}^{2 \mathrm{a}(\xi+\mathrm{C})}-1}{\mathrm{e}^{2 \mathrm{a}(\xi+\mathrm{C})}+1}\right] .
$$

Family 8: When $\mathrm{a}^{2}+\mathrm{b}^{2}=\mathrm{c}^{2}$,

$$
\Phi(\xi)=-2 \arctan \left[\frac{(\mathrm{b}+\mathrm{c})(\mathrm{a}(\xi+\mathrm{C})+2)}{\mathrm{a}^{2}(\xi+\mathrm{C})}\right] .
$$

Family 9: When $\mathrm{a}=\mathrm{b}=\mathrm{c}=\mathrm{ka}$,

$$
\Phi(\xi)=2 \arctan \left[\mathrm{e}^{\mathrm{ka}(\xi+\mathrm{C})}-1\right] .
$$

Family 10: When $\mathrm{a}=\mathrm{c}=\mathrm{ka}$ and $\mathrm{b}=-\mathrm{ka}$,

$$
\Phi(\xi)=-2 \arctan \left[\frac{\mathrm{e}^{\mathrm{ka}(\xi+\mathrm{C})}}{-1+\mathrm{e}^{\mathrm{ka}(\xi+\mathrm{C})}}\right] .
$$

Family 11: When $c=a$,

$$
\Phi(\xi)=-2 \arctan \left[\frac{(\mathrm{a}+\mathrm{b}) \mathrm{e}^{\mathrm{b}(\xi+\mathrm{C})}-1}{(\mathrm{a}-\mathrm{b}) \mathrm{e}^{\mathrm{b}(\xi+\mathrm{C})}-1}\right] .
$$

Family 12: When a $=\mathrm{c}$,

$$
\Phi(\xi)=2 \arctan \left[\frac{(\mathrm{b}+\mathrm{c}) \mathrm{e}^{\mathrm{b}(\xi+\mathrm{C})}+1}{(\mathrm{~b}-\mathrm{c}) \mathrm{e}^{\mathrm{b}(\xi+\mathrm{C})}-1}\right] .
$$

Family 13: When $\mathrm{c}=-\mathrm{a}$,

$$
\Phi(\xi)=2 \arctan \left[\frac{\mathrm{e}^{\mathrm{b}(\xi+\mathrm{C})}+\mathrm{b}-\mathrm{a}}{\mathrm{e}^{\mathrm{b}(\xi+\mathrm{C})}-\mathrm{b}-\mathrm{a}}\right] .
$$


Family 14: When $b=-c$,

$$
\Phi(\xi)=-2 \arctan \left[\frac{\mathrm{ae}^{\mathrm{a}(\xi+\mathrm{C})}}{\mathrm{ce}^{\mathrm{a}(\xi+\mathrm{C})}-1}\right] .
$$

Family 15: When $b=0$ and $a=c$,

$$
\Phi(\xi)=-2 \arctan \left[\frac{\mathrm{c}(\xi+\mathrm{C})+2}{\mathrm{c}(\xi+\mathrm{C})}\right] .
$$

Family 16: When $\mathrm{a}=0$ and $\mathrm{b}=\mathrm{c}$,

$$
\Phi(\xi)=2 \arctan [\mathrm{c}(\xi+\mathrm{C})] .
$$

Family17: When $\mathrm{a}=0$ and $\mathrm{b}=-\mathrm{c}$,

$$
\Phi(\xi)=-2 \arctan \left[\frac{1}{\mathrm{c}(\xi+\mathrm{C})}\right],
$$

where $A_{k}(k=0,2, \ldots, m), B_{k}(k=1,2, \ldots, m), a, b$ and $c$ are constants to be determined later. But, the positive integer $\mathrm{m}$ can be determined by considering the homogeneous balance between the highest order derivatives and nonlinear terms appearing in Eq. (7). If $\mathrm{m}$ is not an integer, then a transformation formula should be used to overcome this difficulty.

Step 3. Substituting (6) into Eq. (5) with the value of $\mathrm{m}$ obtained in Step 2. Collecting the coefficients of $\tan \left(\frac{\Phi(\xi)}{2}\right)^{\mathrm{k}}, \cot \left(\frac{\Phi(\xi)}{2}\right)^{\mathrm{k}}(\mathrm{k}=0,1,2, \ldots)$, then setting each coefficient to zero, we can get a set of over-determined partial differential equations for $\mathrm{A}_{0}, \mathrm{~A}_{\mathrm{k}}(\mathrm{k}=1,2, \ldots, \mathrm{m}), \mathrm{B}_{\mathrm{k}}(\mathrm{k}=1,2, \ldots, \mathrm{m}) \mathrm{a}, \mathrm{b}, \mathrm{c}$ and $\mathrm{p}$ with the aid of symbolic computation Maple 13.

Step 4. Solving the algebraic equations in Step 3, then substituting $A_{0}, A_{1}, B_{1}, \ldots, A_{m}, B_{m}$, $\mu, \mathrm{p}$ in (6).

\section{Illustrative Examples}

In this section, we present several examples to illustrate the applicability of improved $\tan (\Phi(\xi) / 2)$-expansion method to solve nonlinear fractional partial differential equations introduced in "Introduction" section.

\section{The (2+1)-Dimensional Zoomeron Equation}

Consider the nonlinear (2+1)-dimensional Zoomeron equation as follows

$$
\left(\frac{\mathrm{u}_{\mathrm{xy}}}{\mathrm{u}}\right)_{\mathrm{tt}}-\left(\frac{\mathrm{u}_{\mathrm{xy}}}{\mathrm{u}}\right)_{\mathrm{xx}}+2\left(\mathrm{u}^{2}\right)_{\mathrm{xt}}=0,
$$

by using the wave variable $\xi=\mu(\mathrm{x}+\mathrm{y}-\mathrm{mt})$ reduce it to an ODE as follows

$$
\mu^{2}\left(\mathrm{~m}^{2}-1\right) \mathrm{u}^{\prime \prime 3}+\mathrm{Nu}=0,
$$

where obtained by twice integrating and neglecting the constant of integration. Balancing the $\mathrm{u}^{\prime \prime}$ and $\mathrm{u}^{3}$ by using homogenous principal, we have

$$
\mathrm{M}+2=3 \mathrm{M}, \quad \Rightarrow \mathrm{M}=1 .
$$


Then the trail solution is

$$
\mathrm{u}(\xi)=\mathrm{A}_{0}+\mathrm{A}_{1}\left[\mathrm{p}+\tan \left(\frac{\Phi(\xi)}{2}\right)\right]+\mathrm{B}_{1}\left[\mathrm{p}+\tan \left(\frac{\Phi(\xi)}{2}\right)\right]^{-1} .
$$

Substituting (28) and (7) into Eq. (26) and by using the well-known Maple software, we obtain the following sets of non-trivial solutions

Set I:

$$
\begin{gathered}
\mathrm{m}=\mathrm{m}, \quad \mu= \pm \frac{2(\mathrm{~b}-\mathrm{c}) \mathrm{B}_{1}}{\mathrm{a}^{2}+\mathrm{b}^{2}-\mathrm{c}^{2}} \sqrt{\frac{\mathrm{m}}{\mathrm{m}^{2}-1}}, \quad \mathrm{~A}_{0}=0, \quad \mathrm{~A}_{1}=0, \quad \mathrm{~B}_{1}=\mathrm{B}_{1}, \quad \mathrm{p}=-\frac{\mathrm{a}}{\mathrm{b}-\mathrm{c}} \\
\mathrm{N}=\frac{2 \mathrm{~m}(\mathrm{~b}-\mathrm{c})^{2} \mathrm{~B}_{1}^{2}}{\mathrm{a}^{2}+\mathrm{b}^{2}-\mathrm{c}^{2}}, \quad \mathrm{u}(\xi)=\mathrm{B}_{1}\left[-\frac{\mathrm{a}}{\mathrm{b}-\mathrm{c}}+\tan \left(\frac{\Phi(\xi)}{2}\right)\right]^{-1},
\end{gathered}
$$

where a, b and c are arbitrary constants. By using of the (30) and Family 1 and $\mathbf{4}$ respectively can be written as

$$
\begin{aligned}
& \mathrm{u}_{1}(\xi)=-\frac{(\mathrm{b}-\mathrm{c}) \mathrm{B}_{1}}{\sqrt{\mathrm{c}^{2}-\mathrm{b}^{2}-\mathrm{a}^{2}}} \cot \left(\frac{\sqrt{\mathrm{c}^{2}-\mathrm{b}^{2}-\mathrm{a}^{2}}}{2}(\xi+C)\right), \\
& \mathrm{u}_{2}(\xi)=\frac{\mathrm{cB}_{1}}{\sqrt{\mathrm{c}^{2}-\mathrm{a}^{2}}} \cot \left(\frac{\sqrt{\mathrm{c}^{2}-\mathrm{a}^{2}}}{2}(\xi+C)\right) .
\end{aligned}
$$

By using of the (30) and Family $\mathbf{2 , 3}$ and respectively get

$$
\begin{aligned}
& \mathrm{u}_{3}(\xi)=\frac{(\mathrm{b}-\mathrm{c}) \mathrm{B}_{1}}{\sqrt{\mathrm{a}^{2}+\mathrm{b}^{2}-\mathrm{c}^{2}}} \operatorname{coth}\left(\frac{\sqrt{\mathrm{a}^{2}+\mathrm{b}^{2}-\mathrm{c}^{2}}}{2}(\xi+C)\right), \\
& \mathrm{u}_{4}(\xi)=\frac{\mathrm{bB}_{1}}{\sqrt{\mathrm{a}^{2}+\mathrm{b}^{2}}} \operatorname{coth}\left(\frac{\sqrt{\mathrm{a}^{2}+\mathrm{b}^{2}}}{2}(\xi+C)\right), \\
& \mathrm{u}_{5}(\xi)= \pm \mathrm{B}_{1} \sqrt{\frac{\mathrm{b}-\mathrm{c}}{\mathrm{b}+\mathrm{c}}} \operatorname{coth}\left(\frac{\sqrt{\mathrm{b}^{2}-\mathrm{c}^{2}}}{2}(\xi+C)\right) .
\end{aligned}
$$

By using of the (30) and Family 6 we get

$$
\mathrm{u}_{6}(\xi)=\mathrm{B}_{1} \cot \left(\frac{1}{2} \arctan \left[\frac{\mathrm{e}^{2 \mathrm{~b}(\xi+\mathrm{C})}-1}{\mathrm{e}^{2 \mathrm{~b}(\xi+\mathrm{C})}+1}, \frac{2 \mathrm{e}^{\mathrm{b}(\xi+\mathrm{C})}}{\mathrm{e}^{2 \mathrm{~b}(\xi+\mathrm{C})}+1}\right]\right) .
$$

By using of the (30) and Family 10, 11, 12, 13 and 14 respectively give

$$
\begin{aligned}
& \mathrm{u}_{7}(\xi)=\mathrm{B}_{1}\left[\frac{1}{2}-\frac{\mathrm{e}^{\mathrm{ka}(\xi+\mathrm{C})}}{\left[\mathrm{e}^{\mathrm{ka}(\xi+\mathrm{C})}-1\right]}\right]^{-1}, \mathrm{u}_{8}(\xi)=\mathrm{B}_{1}\left[\frac{\mathrm{a}}{\mathrm{a}-\mathrm{b}}-\frac{(\mathrm{a}+\mathrm{b}) \mathrm{e}^{\mathrm{b}(\xi+\mathrm{C})}-1}{(\mathrm{a}-\mathrm{b}) \mathrm{e}^{\mathrm{b}(\xi+\mathrm{C})}-1}\right]^{-1} \\
& \mathrm{u}_{9}(\xi)=\mathrm{B}_{1}\left[\frac{\mathrm{c}}{\mathrm{b}-\mathrm{c}}+\frac{(\mathrm{b}+\mathrm{c}) \mathrm{e}^{\mathrm{b}(\xi+\mathrm{C})}+1}{(\mathrm{~b}-\mathrm{c}) \mathrm{e}^{\mathrm{b}(\xi+\mathrm{C})}-1}\right]^{-1}, \mathrm{u}_{10}(\xi)=\mathrm{B}_{1}\left[-\frac{\mathrm{a}}{\mathrm{a}+\mathrm{b}}+\frac{\mathrm{e}^{\mathrm{b}(\xi+\mathrm{C})}+\mathrm{b}-\mathrm{a}}{\mathrm{e}^{\mathrm{b}(\xi+\mathrm{C})}-\mathrm{b}-\mathrm{a}}\right]^{-1}, \\
& \mathrm{u}_{11}(\xi)=\mathrm{B}_{1}\left[\frac{\mathrm{a}}{2 \mathrm{c}}-\frac{\mathrm{a} \mathrm{e}^{\mathrm{a}(\xi+\mathrm{C})}}{\mathrm{ce}^{\mathrm{a}(\xi+\mathrm{C})}-1}\right]^{-1} .
\end{aligned}
$$


By using of the (29) and Family 15, 16 and $\mathbf{1 7}$ respectively give

$$
\mathrm{u}_{12}(\xi)=-\frac{\mathrm{cB}_{1}}{2}(\xi+\mathrm{C}), \quad \mathrm{u}_{13}(\xi)=\frac{\mathrm{B}_{1}}{\mathrm{c}(\xi+\mathrm{C})}, \quad \mathrm{u}_{14}(\xi)=-\mathrm{B}_{1} \mathrm{c}(\xi+\mathrm{C}),
$$

where $\xi= \pm \frac{2(b-c) B_{1}}{a^{2}+b^{2}-c^{2}} \sqrt{\frac{m}{m^{2}-1}}(x+y-m t)$.

Set II:

$$
\begin{gathered}
\mu=\mu, \quad \mathrm{A}_{0}= \pm \frac{\mu(\mathrm{a}+\mathrm{p}(\mathrm{b}-\mathrm{c}))}{2} \sqrt{\frac{\mathrm{m}^{2}-1}{\mathrm{~m}}}, \mathrm{~A}_{1}=0 \\
\mathrm{~B}_{1}=\mp \frac{\mu\left(\mathrm{p}^{2}(\mathrm{~b}-\mathrm{c})-(\mathrm{b}+\mathrm{c})+2 \mathrm{ap}\right)}{2} \sqrt{\frac{\mathrm{m}^{2}-1}{\mathrm{~m}}} \\
\mathrm{~m}=\mathrm{m}, \mathrm{p}=\mathrm{p}, \mathrm{N}=\frac{\mu^{2}\left(\mathrm{~m}^{2}-1\right)}{2}, \mathrm{u}(\xi)=\mathrm{A}_{0}+\mathrm{B}_{1}\left[\mathrm{p}+\tan \left(\frac{\Phi(\xi)}{2}\right)\right]^{-1},
\end{gathered}
$$

where a, b and c are arbitrary constants. By using of the (37) and Family 1 and $\mathbf{4}$ respectively get to

$$
\begin{aligned}
\mathrm{u}_{15}(\xi)= \pm & \frac{\mu}{2} \sqrt{\frac{\mathrm{m}^{2}-1}{\mathrm{~m}}}\left\{(\mathrm{a}+\mathrm{p}(\mathrm{b}-\mathrm{c}))-\left(\mathrm{p}^{2}(\mathrm{~b}-\mathrm{c})-(\mathrm{b}+\mathrm{c})+2 \mathrm{ap}\right)\right. \\
\times & {\left.\left[\mathrm{p}+\frac{\mathrm{a}}{\mathrm{b}-\mathrm{c}}-\frac{\sqrt{\mathrm{c}^{2}-\mathrm{b}^{2}-\mathrm{a}^{2}}}{\mathrm{~b}-\mathrm{c}} \tan \left(\frac{\sqrt{\mathrm{c}^{2}-\mathrm{b}^{2}-\mathrm{a}^{2}}}{2}(\xi+C)\right)\right]^{-1}\right\} } \\
\mathrm{u}_{16}(\xi)= \pm & \frac{\mu}{2} \sqrt{\frac{\mathrm{m}^{2}-1}{\mathrm{~m}}}\left\{(\mathrm{a}-\mathrm{pc})-\left(-\mathrm{p}^{2} \mathrm{c}-\mathrm{c}+2 \mathrm{ap}\right)\right. \\
\times & {\left.\left[\mathrm{p}-\frac{\mathrm{a}}{\mathrm{c}}+\frac{\sqrt{\mathrm{c}^{2}-\mathrm{a}^{2}}}{\mathrm{~b}} \tanh \left(\frac{\sqrt{\mathrm{c}^{2}-\mathrm{a}^{2}}}{2}(\xi+C)\right)\right]^{-1}\right\} }
\end{aligned}
$$

By using of the (37) and Family $\mathbf{2 , 3}$ and $\mathbf{5}$ respectively can be written as

$$
\begin{aligned}
\mathrm{u}_{17}(\xi)= \pm & \frac{\mu}{2} \sqrt{\frac{\mathrm{m}^{2}-1}{\mathrm{~m}}}\left\{(\mathrm{a}+\mathrm{p}(\mathrm{b}-\mathrm{c}))-\left(\mathrm{p}^{2}(\mathrm{~b}-\mathrm{c})-(\mathrm{b}+\mathrm{c})+2 \mathrm{ap}\right)\right. \\
\times & {\left.\left[\mathrm{p}+\frac{\mathrm{a}}{\mathrm{b}-\mathrm{c}}+\frac{\sqrt{\mathrm{a}^{2}+\mathrm{b}^{2}-\mathrm{c}^{2}}}{\mathrm{~b}-\mathrm{c}} \tanh \left(\frac{\sqrt{\mathrm{a}^{2}+\mathrm{b}^{2}-\mathrm{c}^{2}}}{2}(\xi+C)\right)\right]^{-1}\right\}, } \\
\mathrm{u}_{18}(\xi)= \pm & \frac{\mu}{2} \sqrt{\frac{\mathrm{m}^{2}-1}{\mathrm{~m}}}\left\{(\mathrm{a}+\mathrm{pb})-\left(\mathrm{p}^{2} \mathrm{~b}-\mathrm{b}+2 \mathrm{ap}\right)\right. \\
\times & {\left.\left[\mathrm{p}+\frac{\mathrm{a}}{\mathrm{b}}+\frac{\sqrt{\mathrm{a}^{2}+\mathrm{b}^{2}}}{\mathrm{~b}} \tanh \left(\frac{\sqrt{\mathrm{a}^{2}+\mathrm{b}^{2}}}{2}(\xi+C)\right)\right]^{-1}\right\}, }
\end{aligned}
$$




$$
\begin{aligned}
\mathrm{u}_{19}(\xi)= \pm & \frac{\mu}{2} \sqrt{\frac{\mathrm{m}^{2}-1}{\mathrm{~m}}}\left\{\mathrm{p}(\mathrm{b}-\mathrm{c})-\left(\mathrm{p}^{2}(\mathrm{~b}-\mathrm{c})-(\mathrm{b}+\mathrm{c})\right)\right. \\
\times & {\left.\left[\mathrm{p}+\frac{\sqrt{\mathrm{b}^{2}-\mathrm{c}^{2}}}{\mathrm{~b}-\mathrm{c}} \tanh \left(\frac{\sqrt{\mathrm{c}^{2}-\mathrm{a}^{2}}}{2}(\xi+\mathrm{C})\right)\right]^{-1}\right\} . }
\end{aligned}
$$

By using of the (30) and Family 6, 7 and respectively can be written as

$$
\begin{aligned}
\mathrm{u}_{20}(\xi)= \pm & \frac{\mu}{2} \sqrt{\frac{\mathrm{m}^{2}-1}{\mathrm{~m}}} \\
& \times\left\{\mathrm{pb}-\mathrm{b}\left(\mathrm{p}^{2}-1\right)\left[\mathrm{p}+\tan \left(\frac{1}{2} \arctan \left[\frac{\mathrm{e}^{2 \mathrm{~b}(\xi+\mathrm{C})}-1}{\mathrm{e}^{2 \mathrm{~b}(\xi+\mathrm{C})}+1}, \frac{2 \mathrm{e}^{\mathrm{b}(\xi+\mathrm{C})}}{\mathrm{e}^{2 \mathrm{~b}(\xi+\mathrm{C})}+1}\right]\right)\right]^{-1}\right\}, \\
\mathrm{u}_{21}(\xi)= \pm & \frac{\mu}{2} \sqrt{\frac{\mathrm{m}^{2}-1}{\mathrm{~m}}}\left\{\mathrm{a}-2 \mathrm{ap}\left[\mathrm{p}+\tan \left(\frac{1}{2} \arctan \left[\frac{2 \mathrm{e}^{\mathrm{a}(\xi+C)}}{\mathrm{e}^{2 \mathrm{a}(\xi+\mathrm{C})}+1}, \frac{\mathrm{e}^{2 \mathrm{a}(\xi+\mathrm{C})}-1}{\mathrm{e}^{2 \mathrm{a}(\xi+\mathrm{C})}+1}\right]\right)\right]^{-1}\right\}, \\
\mathrm{u}_{22}(\xi)= \pm & \frac{\mu}{2} \sqrt{\frac{\mathrm{m}^{2}-1}{\mathrm{~m}}}\left\{\left(\sqrt{\mathrm{c}^{2}-\mathrm{b}^{2}}+\mathrm{p}(\mathrm{b}-\mathrm{c})\right)-\left(\mathrm{p}^{2}(\mathrm{~b}-\mathrm{c})-(\mathrm{b}+\mathrm{c})\right.\right. \\
& \left.\left.+2 \mathrm{p} \sqrt{\mathrm{c}^{2}-\mathrm{b}^{2}}\right)\left[\mathrm{p}+\frac{2+\mathrm{a} \xi}{(\mathrm{b}-\mathrm{c}) \xi}\right]^{-1}\right\} .
\end{aligned}
$$

By using of the (30) and Family 9, 10, 11, 12, 13 and 14 respectively can be written as

$$
\begin{aligned}
& \mathrm{u}_{23}(\xi)= \pm \frac{\mu}{2} \sqrt{\frac{\mathrm{m}^{2}-1}{\mathrm{~m}}}\left\{\mathrm{ak}-2 \mathrm{ak}(\mathrm{p}-1)\left[\mathrm{p}-1+\mathrm{e}^{\mathrm{ak}(\xi+\mathrm{C})}\right]^{-1}\right\}, \\
& \mathrm{u}_{24}(\xi)= \pm \frac{\mu}{2} \sqrt{\frac{\mathrm{m}^{2}-1}{\mathrm{~m}}}\left\{\mathrm{ak}(1-2 \mathrm{p})+2 \mathrm{akp}(\mathrm{p}-1)\left[\mathrm{p}-\frac{\mathrm{e}^{\mathrm{ak}(\xi+\mathrm{C})}}{-1+\mathrm{e}^{\mathrm{ak}(\xi+\mathrm{C})}}\right]^{-1}\right\}, \\
& \mathrm{u}_{25}(\xi)= \pm \frac{\mu}{2} \sqrt{\frac{\mathrm{m}^{2}-1}{\mathrm{~m}}}\left\{(\mathrm{a}+\mathrm{p}(\mathrm{b}-\mathrm{a}))-\left(\mathrm{p}^{2}(\mathrm{~b}-\mathrm{a})-(\mathrm{a}+\mathrm{b})+2 \mathrm{ap}\right)\right. \\
& \left.\times\left[p-\frac{(a+b) e^{b(\xi+C)}-1}{(a-b) e^{b(\xi+C)}-1}\right]^{-1}\right\} \\
& \mathrm{u}_{26}(\xi)= \pm \frac{\mu}{2} \sqrt{\frac{\mathrm{m}^{2}-1}{\mathrm{~m}}}\left\{(\mathrm{c}+\mathrm{p}(\mathrm{b}-\mathrm{c}))-\left(\mathrm{p}^{2}(\mathrm{~b}-\mathrm{c})-(\mathrm{b}+\mathrm{c})+2 \mathrm{cp}\right)\right. \\
& \left.\times\left[p+\frac{(b+c) e^{b(\xi+C)}+1}{(b-c) e^{b(\xi+C)}-1}\right]^{-1}\right\} \\
& \mathrm{u}_{27}(\xi)= \pm \frac{\mu}{2} \sqrt{\frac{\mathrm{m}^{2}-1}{\mathrm{~m}}}\left\{(-\mathrm{a}+\mathrm{p}(\mathrm{b}+\mathrm{a}))-\left(\mathrm{p}^{2}(\mathrm{~b}+\mathrm{a})-(\mathrm{b}-\mathrm{a})+2 \mathrm{cp}\right)\right.
\end{aligned}
$$




$$
\begin{gathered}
\left.\times\left[\mathrm{p}+\frac{\mathrm{e}^{\mathrm{b}(\xi+\mathrm{C})}+\mathrm{b}-\mathrm{a}}{\mathrm{e}^{\mathrm{b}(\xi+\mathrm{C})}-\mathrm{b}-\mathrm{a}}\right]^{-1}\right\}, \\
\mathrm{u}_{28}(\xi)= \pm \frac{\mu}{2} \sqrt{\frac{\mathrm{m}^{2}-1}{\mathrm{~m}}}\left\{(\mathrm{a}-2 \mathrm{pc})-\left(-2 \mathrm{cp}^{2}+2 \mathrm{ap}\right)\left[\mathrm{p}-\frac{\mathrm{ae^{ \textrm {a } ( \xi + C ) }}}{\mathrm{ce} \mathrm{e}^{\mathrm{a}(\xi+\mathrm{C})}-1}\right]^{-1}\right\} .
\end{gathered}
$$

By using of the (29) and Family 15, 16 and $\mathbf{1 7}$ respectively can be written as

$$
\begin{aligned}
& \mathrm{u}_{29}(\xi)= \pm \frac{\mu}{2} \sqrt{\frac{\mathrm{m}^{2}-1}{\mathrm{~m}}}\left\{(\mathrm{c}-\mathrm{pc})-\left(-\mathrm{cp}^{2}-\mathrm{c}+2 \mathrm{cp}\right)\left[\mathrm{p}-\frac{2+\mathrm{c}(\xi+\mathrm{C})}{\mathrm{c}(\xi+\mathrm{C})}\right]^{-1}\right\}, \\
& \mathrm{u}_{30}(\xi)= \pm \frac{\mu}{2} \sqrt{\frac{\mathrm{m}^{2}-1}{\mathrm{~m}}}\left\{2 \mathrm{c}[\mathrm{p}+\mathrm{c}(\xi+\mathrm{C})]^{-1}\right\} \\
& \mathrm{u}_{31}(\xi)= \pm \frac{\mu}{2} \sqrt{\frac{\mathrm{m}^{2}-1}{\mathrm{~m}}}\left\{-2 \mathrm{pc}+2 \mathrm{pc}\left[\mathrm{p}-\frac{1}{\mathrm{c}(\xi+\mathrm{C})}\right]^{-1}\right\},
\end{aligned}
$$

where $\xi=\mu(\mathrm{x}+\mathrm{y}-\mathrm{mt})$.

\section{Set III:}

$$
\begin{aligned}
\mathrm{m}= & \mathrm{m}, \quad \mu= \pm \frac{2(\mathrm{~b}-\mathrm{c}) \mathrm{B}_{1}}{\mathrm{a}^{2}+\mathrm{b}^{2}-\mathrm{c}^{2}} \sqrt{\frac{\mathrm{m}}{\mathrm{m}^{2}-1}}, \quad \mathrm{~A}_{0}=0, \quad \mathrm{~A}_{1}=-\frac{(\mathrm{b}-\mathrm{c})^{2} \mathrm{~B}_{1}}{\mathrm{a}^{2}+\mathrm{b}^{2}-\mathrm{c}^{2}}, \quad \mathrm{~B}_{1}=\mathrm{B}_{1}, \\
\mathrm{p}= & -\frac{\mathrm{a}}{\mathrm{b}-\mathrm{c}}, \\
\mathrm{N}= & -\frac{4 \mathrm{~m}(\mathrm{~b}-\mathrm{c})^{2} \mathrm{~B}_{1}^{2}}{\mathrm{a}^{2}+\mathrm{b}^{2}-\mathrm{c}^{2}}, \quad \mathrm{u}(\xi)=\mathrm{A}_{1}\left[-\frac{\mathrm{a}}{\mathrm{b}-\mathrm{c}}+\tan \left(\frac{\Phi(\xi)}{2}\right)\right] \\
& +\mathrm{B}_{1}\left[-\frac{\mathrm{a}}{\mathrm{b}-\mathrm{c}}+\tan \left(\frac{\Phi(\xi)}{2}\right)\right]^{-1},
\end{aligned}
$$

where a, b and c are arbitrary constants. By using of the (44) and Family 1 and $\mathbf{4}$ respectively get to

$$
\begin{aligned}
& \mathrm{u}_{32}(\xi)=\frac{(\mathrm{b}-\mathrm{c}) \mathrm{B}_{1}}{\sqrt{\mathrm{c}^{2}-\mathrm{b}^{2}-\mathrm{a}^{2}}}\left[\tan \left(\frac{\sqrt{\mathrm{c}^{2}-\mathrm{b}^{2}-\mathrm{a}^{2}}}{2}(\xi+\mathrm{C})\right)-\cot \left(\frac{\sqrt{\mathrm{c}^{2}-\mathrm{b}^{2}-\mathrm{a}^{2}}}{2}(\xi+\mathrm{C})\right)\right], \\
& \mathrm{u}_{33}(\xi)=\frac{-\mathrm{cB} 1}{\sqrt{\mathrm{c}^{2}-\mathrm{a}^{2}}}\left[\tan \left(\frac{\sqrt{\mathrm{c}^{2}-\mathrm{a}^{2}}}{2}(\xi+\mathrm{C})\right)-\cot \left(\frac{\sqrt{\mathrm{c}^{2}-\mathrm{a}^{2}}}{2}(\xi+\mathrm{C})\right)\right] .
\end{aligned}
$$

By using of the (44) and Family $\mathbf{2 , 3}$ and $\mathbf{5}$ can be written as

$$
\begin{aligned}
& \mathrm{u}_{34}(\xi)=\frac{(\mathrm{b}-\mathrm{c}) \mathrm{B}_{1}}{\sqrt{\mathrm{a}^{2}+\mathrm{b}^{2}-\mathrm{c}^{2}}}\left[\tanh \left(\frac{\sqrt{\mathrm{a}^{2}+\mathrm{b}^{2}-\mathrm{c}^{2}}}{2}(\xi+C)\right)+\operatorname{coth}\left(\frac{\sqrt{\mathrm{a}^{2}+\mathrm{b}^{2}-\mathrm{c}^{2}}}{2}(\xi+C)\right)\right], \\
& \mathrm{u}_{35}(\xi)=\frac{\mathrm{bB}_{1}}{\sqrt{\mathrm{a}^{2}+\mathrm{b}^{2}}}\left[\tanh \left(\frac{\sqrt{\mathrm{a}^{2}+\mathrm{b}^{2}}}{2}(\xi+C)\right)+\operatorname{coth}\left(\frac{\sqrt{\mathrm{a}^{2}+\mathrm{b}^{2}}}{2}(\xi+C)\right)\right], \\
& \mathrm{u}_{36}(\xi)=-\mathrm{B}_{1} \sqrt{\frac{\mathrm{b}-\mathrm{c}}{\mathrm{b}+\mathrm{c}}}\left[\tanh \left(\frac{\sqrt{\mathrm{b}^{2}-\mathrm{c}^{2}}}{2}(\xi+C)\right)-\operatorname{coth}\left(\frac{\sqrt{\mathrm{b}^{2}-\mathrm{c}^{2}}}{2}(\xi+C)\right)\right] .
\end{aligned}
$$


By using of the (44) and Family 6 we get

$$
\begin{aligned}
\mathrm{u}_{37}(\xi)= & \mathrm{B}_{1}\left[\cot \left(\frac{1}{2} \arctan \left[\frac{\mathrm{e}^{2 \mathrm{~b}(\xi+\mathrm{C})}-1}{\mathrm{e}^{2 \mathrm{~b}(\xi+\mathrm{C})}+1}, \frac{2 \mathrm{e}^{\mathrm{b}(\xi+\mathrm{C})}}{\mathrm{e}^{2 \mathrm{~b}(\xi+\mathrm{C})}+1}\right]\right)\right. \\
& \left.-\tan \left(\frac{1}{2} \arctan \left[\frac{\mathrm{e}^{2 \mathrm{~b}(\xi+\mathrm{C})}-1}{\mathrm{e}^{2 \mathrm{~b}(\xi+\mathrm{C})}+1}, \frac{2 \mathrm{e}^{\mathrm{b}(\xi+\mathrm{C})}}{\mathrm{e}^{2 \mathrm{~b}(\xi+\mathrm{C})}+1}\right]\right)\right] .
\end{aligned}
$$

By using of the (44) and Family 10, 11, 12, 13 and 14 respectively can be written as

$$
\begin{aligned}
& \mathrm{u}_{38}(\xi)=-4 \mathrm{~B}_{1}\left[\frac{1}{2}-\frac{\mathrm{e}^{\mathrm{ka}(\xi+\mathrm{C})}}{\mathrm{e}^{\mathrm{ka}(\xi+\mathrm{C})}-1}\right]+\mathrm{B}_{1}\left[\frac{1}{2}-\frac{\mathrm{e}^{\mathrm{ka}(\xi+\mathrm{C})}}{\mathrm{e}^{\mathrm{ka}(\xi+\mathrm{C})}-1}\right]^{-1}, \\
& \mathrm{u}_{39}(\xi)=-\frac{(\mathrm{b}-\mathrm{a})^{2}}{\mathrm{~b}^{2}} \mathrm{~B}_{1}\left[\frac{\mathrm{a}}{\mathrm{a}-\mathrm{b}}-\frac{(\mathrm{a}+\mathrm{b}) \mathrm{e}^{\mathrm{b}(\xi+\mathrm{C})}-1}{(\mathrm{a}-\mathrm{b}) \mathrm{e}^{\mathrm{b}(\xi+\mathrm{C})}-1}\right]+\mathrm{B}_{1}\left[\frac{\mathrm{a}}{\mathrm{a}-\mathrm{b}}-\frac{(\mathrm{a}+\mathrm{b}) \mathrm{e}^{\mathrm{b}(\xi+\mathrm{C})}-1}{(\mathrm{a}-\mathrm{b}) \mathrm{e}^{\mathrm{b}(\xi+\mathrm{C})}-1}\right]^{-1}, \\
& \mathrm{u}_{40}(\xi)=-\frac{(\mathrm{b}-\mathrm{c})^{2}}{\mathrm{~b}^{2}} \mathrm{~B}_{1}\left[\frac{\mathrm{c}}{\mathrm{b}-\mathrm{c}}+\frac{(\mathrm{b}+\mathrm{c}) \mathrm{e}^{\mathrm{b}(\xi+\mathrm{C})}+1}{(\mathrm{~b}-\mathrm{c}) \mathrm{e}^{\mathrm{b}(\xi+\mathrm{C})}-1}\right]+\mathrm{B}_{1}\left[\frac{\mathrm{c}}{\mathrm{b}-\mathrm{c}}+\frac{(\mathrm{b}+\mathrm{c}) \mathrm{e}^{\mathrm{b}(\xi+\mathrm{C})}+1}{(\mathrm{~b}-\mathrm{c}) \mathrm{e}^{\mathrm{b}(\xi+\mathrm{C})}-1}\right]^{-1}, \\
& \mathrm{u}_{41}(\xi)=-\frac{(\mathrm{b}+\mathrm{a})^{2}}{\mathrm{~b}^{2}} \mathrm{~B}_{1}\left[-\frac{\mathrm{a}}{\mathrm{a}+\mathrm{b}}+\frac{\mathrm{e}^{\mathrm{b}(\xi+\mathrm{C})}+\mathrm{b}-\mathrm{a}}{\mathrm{e}^{\mathrm{b}(\xi+\mathrm{C})}-\mathrm{b}-\mathrm{a}}\right]+\mathrm{B}_{1}\left[-\frac{\mathrm{a}}{\mathrm{a}+\mathrm{b}}+\frac{\mathrm{e}^{\mathrm{b}(\xi+C)}+\mathrm{b}-\mathrm{a}}{\mathrm{e}^{\mathrm{b}(\xi+C)}-\mathrm{b}-\mathrm{a}}\right]^{-1}, \\
& \mathrm{u}_{42}(\xi)=-\frac{4 \mathrm{c}^{2}}{\mathrm{~b}^{2}} \mathrm{~B}_{1}\left[\frac{\mathrm{a}}{2 \mathrm{c}}-\frac{\mathrm{a} \mathrm{e}^{\mathrm{a}(\xi+C)}}{\mathrm{ce} \mathrm{e}^{\mathrm{a}(\xi+\mathrm{C})}-1}\right]+\mathrm{B}_{1}\left[\frac{\mathrm{a}}{2 \mathrm{c}}-\frac{\mathrm{a} \mathrm{e}^{\mathrm{a}(\xi+C)}}{\mathrm{ce} \mathrm{e}^{\mathrm{a}(\xi+\mathrm{C})}-1}\right]^{-1},
\end{aligned}
$$

where $\xi= \pm \frac{2(b-c) B_{1}}{a^{2}+b^{2}-c^{2}} \sqrt{\frac{m}{m^{2}-1}}(x+y-m t)$.

Set IV:

$$
\begin{aligned}
& \mu= \pm \frac{2 \mathrm{~A}_{1}}{\mathrm{~b}-\mathrm{c}} \sqrt{\frac{\mathrm{m}^{2}-1}{\mathrm{~m}}}, \quad \mathrm{~A}_{0}=-\frac{\mathrm{A}_{1}(\mathrm{a}+\mathrm{p}(\mathrm{b}-\mathrm{c}))}{\mathrm{b}-\mathrm{c}}, \quad \mathrm{A}_{1}=\mathrm{A}_{1}, \quad \mathrm{~B}_{1}=0, \\
& \mathrm{~m}=\mathrm{m}, \quad \mathrm{p}=\mathrm{p}, \quad \mathrm{N}=\frac{2\left(\mathrm{a}^{2}+\mathrm{b}^{2}-\mathrm{c}^{2}\right) \mathrm{mA}_{1}^{2}}{(\mathrm{~b}-\mathrm{c})^{2}}, \quad \mathrm{u}(\xi)=\mathrm{A}_{0}+\mathrm{A}_{1} \mathrm{p}+\mathrm{A}_{1} \tan \left(\frac{\Phi(\xi)}{2}\right),
\end{aligned}
$$

where a, b and c are arbitrary constants. By using of the (50) and Family $\mathbf{1}$ and $\mathbf{4}$ respectively get to

$$
\begin{aligned}
& \mathrm{u}_{43}(\xi)=-\frac{\mathrm{A}_{1} \sqrt{\mathrm{c}^{2}-\mathrm{b}^{2}-\mathrm{a}^{2}}}{\mathrm{~b}-\mathrm{c}} \tan \left(\frac{\sqrt{\mathrm{c}^{2}-\mathrm{b}^{2}-\mathrm{a}^{2}}}{2}(\xi+C)\right), \\
& \mathrm{u}_{44}(\xi)=\frac{\mathrm{A}_{1} \sqrt{\mathrm{c}^{2}-\mathrm{a}^{2}}}{\mathrm{c}} \tan \left(\frac{\sqrt{\mathrm{c}^{2}-\mathrm{a}^{2}}}{2}(\xi+C)\right) .
\end{aligned}
$$


By using of the (50) and Family 2, 3 and Family 5 respectively can be written

$$
\begin{aligned}
& \mathrm{u}_{45}(\xi)=\frac{A_{1} \sqrt{a^{2}+b^{2}-c^{2}}}{b-c} \tanh \left(\frac{\sqrt{a^{2}+b^{2}-c^{2}}}{2}(\xi+C)\right) . \\
& u_{46}(\xi)=\frac{A_{1} \sqrt{a^{2}+b^{2}}}{b} \tanh \left(\frac{\sqrt{a^{2}+b^{2}}}{2}(\xi+C)\right) \\
& u_{47}(\xi)=\frac{A_{1} \sqrt{b^{2}-c^{2}}}{b-c} \tanh \left(\frac{\sqrt{b^{2}-c^{2}}}{2}(\xi+C)\right)
\end{aligned}
$$

By using of the (50) and Family $\mathbf{6}$ and $\mathbf{8}$ respectively get to

$$
\begin{aligned}
& \mathrm{u}_{48}(\xi)=\mathrm{A}_{1} \tan \left(\frac{1}{2} \arctan \left[\frac{\mathrm{e}^{2 \mathrm{~b}(\xi+\mathrm{C})}-1}{\mathrm{e}^{2 \mathrm{~b}(\xi+C)}+1}, \frac{2 \mathrm{e}^{\mathrm{b}(\xi+\mathrm{C})}}{\mathrm{e}^{2 \mathrm{~b}(\xi+\mathrm{C})}+1}\right]\right), \\
& \mathrm{u}_{49}(\xi)=-\frac{\mathrm{A}_{1}}{\mathrm{~b}-\mathrm{c}}\left[2-\sqrt{\mathrm{c}^{2}-\mathrm{b}^{2}}(1-(\xi+\mathrm{C}))\right] .
\end{aligned}
$$

By using of the (50) and Family 10, 11, 12, 13 and 14 respectively can be written as

$$
\begin{aligned}
& \mathrm{u}_{50}(\xi)=\frac{1}{2}-\frac{\mathrm{A}_{1} \mathrm{e}^{\mathrm{ak}(\xi+\mathrm{C})}}{-1+\mathrm{e}^{\mathrm{ak}(\xi+\mathrm{C})}}, \quad \mathrm{u}_{51}(\xi)=\frac{\mathrm{aA}_{1}}{\mathrm{a}-\mathrm{b}}-\mathrm{A}_{1} \frac{(\mathrm{a}+\mathrm{b}) \mathrm{e}^{\mathrm{b}(\xi+\mathrm{C})}-1}{(\mathrm{a}-\mathrm{b}) \mathrm{e}^{\mathrm{b}(\xi+\mathrm{C})}-1} \\
& \mathrm{u}_{52}(\xi)=-\frac{\mathrm{cA}_{1}}{\mathrm{~b}-\mathrm{c}}+\mathrm{A}_{1} \frac{(\mathrm{b}+\mathrm{c}) \mathrm{e}^{\mathrm{b}(\xi+\mathrm{C})}+1}{(\mathrm{~b}-\mathrm{c}) \mathrm{e}^{\mathrm{b}(\xi+\mathrm{C})}-1}, \mathrm{u}_{53}(\xi)=-\frac{\mathrm{aA}_{1}}{\mathrm{~b}+\mathrm{a}}+\mathrm{A}_{1} \frac{\mathrm{e}^{\mathrm{b}(\xi+C)}+\mathrm{b}-\mathrm{a}}{\mathrm{e}^{\mathrm{b}(\xi+C)}-\mathrm{b}-\mathrm{a}}, \\
& \mathrm{u}_{54}(\xi)=\frac{\mathrm{aA}_{1}}{2 \mathrm{c}}-\mathrm{A}_{1} \frac{\mathrm{a} \mathrm{e}^{\mathrm{a}(\xi+\mathrm{C})}}{\mathrm{ce}^{\mathrm{a}(\xi+\mathrm{C})}-1}
\end{aligned}
$$

By using of the (49) and Family 15 we get

$$
\mathrm{u}_{55}(\xi)=-\frac{2 \mathrm{~A}_{1}}{\mathrm{c}(\xi+\mathrm{C})},
$$

where $\xi= \pm \frac{2 \mathrm{~A}_{1}}{\mathrm{~b}-\mathrm{c}} \sqrt{\frac{\mathrm{m}^{2}-1}{\mathrm{~m}}}(\mathrm{x}+\mathrm{y}-\mathrm{mt})$.

\section{The Duffing Equation}

Consider the nonlinear Duffing equation as follows

$$
\mathrm{u}_{\mathrm{tt}}+\alpha \mathrm{u}+\beta \mathrm{u}^{3}=0,
$$

by using the wave variable $\xi=\mu(\mathrm{x}+\mathrm{y}-\mathrm{mt})$ reduce it to an ODE as follows

$$
-\mu^{2} \mathrm{~m}^{2} \mathrm{u}^{\prime \prime 3}=0
$$

Balancing the $\mathrm{u}^{\prime \prime}$ and $\mathrm{u}^{3}$ by using homogenous principal, we have

$$
\mathrm{M}+2=3 \mathrm{M}, \quad \Rightarrow \mathrm{M}=1 .
$$


Then the trail solution is

$$
\mathrm{u}(\xi)=\mathrm{A}_{0}+\mathrm{A}_{1}\left[\mathrm{p}+\tan \left(\frac{\Phi(\xi)}{2}\right)\right]+\mathrm{B}_{1}\left[\mathrm{p}+\tan \left(\frac{\Phi(\xi)}{2}\right)\right]^{-1} .
$$

Substituting (59) and (7) into Eq. (57) and by using the well-known Maple software, we obtain the following sets of non-trivial solutions

\section{Set I:}

$$
\begin{aligned}
& \mu=\mu, \quad \mathrm{m}= \pm \frac{1}{\mu} \sqrt{\frac{-2 \alpha}{\mathrm{a}^{2}+\mathrm{b}^{2}-\mathrm{c}^{2}}}, \quad \mathrm{~A}_{0}=0, \quad \mathrm{~A}_{1}=0, \\
& \mathrm{~B}_{1}= \pm \frac{1}{\mathrm{~b}-\mathrm{c}} \sqrt{\frac{-\alpha}{\beta}\left(\mathrm{a}^{2}+\mathrm{b}^{2}-\mathrm{c}^{2}\right)} \\
& \mathrm{p}=-\frac{\mathrm{a}}{\mathrm{b}-\mathrm{c}}, \quad \mathrm{u}(\xi)=\mathrm{B}_{1}\left[-\frac{\mathrm{a}}{\mathrm{b}-\mathrm{c}}+\tan \left(\frac{\Phi(\xi)}{2}\right)\right]^{-1},
\end{aligned}
$$

where a, b and c are arbitrary constants. By using of the (61) and Family $\mathbf{1 ,} 4$ respectively get to

$$
\mathrm{u}_{1}(\xi)=\mp \sqrt{\frac{\alpha}{\beta}} \cot \left(\frac{\sqrt{\mathrm{c}^{2}-\mathrm{b}^{2}-\mathrm{a}^{2}}}{2}(\xi+\mathrm{C})\right), \quad \mathrm{u}_{2}(\xi)= \pm \sqrt{\frac{\alpha}{\beta}} \cot \left(\frac{\sqrt{\mathrm{c}^{2}-\mathrm{a}^{2}}}{2}(\xi+\mathrm{C})\right) .
$$

By using of the (61) and Family 2, 3 and $\mathbf{5}$ respectively get to

$$
\begin{aligned}
& \mathrm{u}_{3}(\xi)= \pm \sqrt{\frac{\alpha}{\beta}} \operatorname{coth}\left(\frac{\sqrt{\mathrm{a}^{2}+\mathrm{b}^{2}-\mathrm{c}^{2}}}{2}(\xi+\mathrm{C})\right), \\
& \mathrm{u}_{4}(\xi)= \pm \sqrt{\frac{\alpha}{\beta}} \operatorname{coth}\left(\frac{\sqrt{\mathrm{a}^{2}+\mathrm{b}^{2}}}{2}(\xi+\mathrm{C})\right), \\
& \mathrm{u}_{5}(\xi)= \pm \sqrt{\frac{\alpha}{\beta}} \operatorname{coth}\left(\frac{\sqrt{\mathrm{b}^{2}-\mathrm{c}^{2}}}{2}(\xi+\mathrm{C})\right) .
\end{aligned}
$$

By using of the (61) and Family 6 we get

$$
\mathrm{u}_{6}(\xi)= \pm \sqrt{\frac{-\alpha}{\beta}} \cot \left(\frac{1}{2} \arctan \left[\frac{\mathrm{e}^{2 \mathrm{~b}(\xi+\mathrm{C})}-1}{\mathrm{e}^{2 \mathrm{~b}(\xi+\mathrm{C})}+1}, \frac{2 \mathrm{e}^{\mathrm{b}(\xi+\mathrm{C})}}{\mathrm{e}^{2 \mathrm{~b}(\xi+\mathrm{C})}+1}\right]\right) .
$$

By using of the (61) and Family 10, 11, 12, 13 and 14 respectively can be written as

$$
\begin{aligned}
& \mathrm{u}_{7}(\xi)=\mp \sqrt{\frac{-\alpha}{\beta}}\left[\frac{1}{2}-\frac{\mathrm{e}^{\mathrm{ka}(\xi+\mathrm{C})}}{\left[\mathrm{e}^{\mathrm{ka}(\xi+\mathrm{C})}-1\right]}\right]^{-1}, \\
& \mathrm{u}_{8}(\xi)= \pm \frac{\mathrm{b}}{\mathrm{b}-\mathrm{a}} \sqrt{\frac{-\alpha}{\beta}}\left[\frac{\mathrm{a}}{\mathrm{a}-\mathrm{b}}-\frac{(\mathrm{a}+\mathrm{b}) \mathrm{e}^{\mathrm{b}(\xi+\mathrm{C})}-1}{(\mathrm{a}-\mathrm{b}) \mathrm{e}^{\mathrm{b}(\xi+\mathrm{C})}-1}\right]^{-1}, \\
& \mathrm{u}_{9}(\xi)=\mp \frac{\mathrm{b}}{\mathrm{b}-\mathrm{c}} \sqrt{\frac{-\alpha}{\beta}}\left[\frac{\mathrm{c}}{\mathrm{b}-\mathrm{c}}-\frac{(\mathrm{b}+\mathrm{c}) \mathrm{e}^{\mathrm{b}(\xi+\mathrm{C})}+1}{(\mathrm{~b}-\mathrm{c}) \mathrm{e}^{\mathrm{b}(\xi+\mathrm{C})}-1}\right]^{-1},
\end{aligned}
$$




$$
\begin{aligned}
& \mathrm{u}_{10}(\xi)=\mp \frac{\mathrm{ab}}{\mathrm{b}+\mathrm{a}} \sqrt{\frac{-\alpha}{\beta}}\left[\frac{\mathrm{a}}{\mathrm{a}+\mathrm{b}}-\frac{\mathrm{e}^{\mathrm{b}(\xi+\mathrm{C})}+\mathrm{b}-\mathrm{a}}{\mathrm{e}^{\mathrm{b}(\xi+\mathrm{C})}-\mathrm{b}-\mathrm{a}}\right]^{-1}, \\
& \mathrm{u}_{11}(\xi)=\mp \frac{\mathrm{a}^{2}}{2 \mathrm{c}} \sqrt{\frac{-\alpha}{\beta}}\left[\frac{\mathrm{a}}{2 \mathrm{c}}-\frac{\mathrm{a} \mathrm{e}^{\mathrm{a}(\xi+\mathrm{C})}}{\mathrm{ce}^{\mathrm{a}(\xi+\mathrm{C})}-1}\right]^{-1},
\end{aligned}
$$

where $\xi=\mu\left(\mathrm{x}+\mathrm{y} \mp \frac{1}{\mu} \sqrt{\frac{-2 \alpha}{\mathrm{a}^{2}+\mathrm{b}^{2}-\mathrm{c}^{2}}} \mathrm{t}\right)$.

Set II:

$$
\begin{aligned}
& \mathrm{m}= \pm \frac{1}{\mu} \sqrt{\frac{-2 \alpha}{\mathrm{a}^{2}+\mathrm{b}^{2}-\mathrm{c}^{2}}}, \quad \mathrm{~A}_{0}= \pm \sqrt{-\frac{\alpha}{3 \beta}}, \quad \mathrm{A}_{1}=0 \\
& \mathrm{~B}_{1}= \pm \frac{2}{3(\mathrm{~b}-\mathrm{c})} \sqrt{\frac{-\alpha}{\beta}\left(\mathrm{a}^{2}+\mathrm{b}^{2}-\mathrm{c}^{2}\right)}, \\
& \mu=\mu, \quad \mathrm{p}=-\frac{\mathrm{a}}{\mathrm{b}-\mathrm{c}}+\frac{\sqrt{\mathrm{a}^{2}+\mathrm{b}^{2}-\mathrm{c}^{2}}}{\sqrt{3}(\mathrm{~b}-\mathrm{c})}, \quad \mathrm{u}(\xi)=\mathrm{A}_{0}+\mathrm{B}_{1}\left[\mathrm{p}+\tan \left(\frac{\Phi(\xi)}{2}\right)\right]^{-1},
\end{aligned}
$$

where a, b and c are arbitrary constants. By using of the (67) and Family $\mathbf{1 ,} 4$ respectively get to

$$
\begin{aligned}
\mathrm{u}_{12}(\xi)= \pm & \sqrt{-\frac{\alpha}{3 \beta}} \pm \frac{2}{3} \sqrt{\frac{-\alpha}{\beta}\left(\mathrm{a}^{2}+\mathrm{b}^{2}-\mathrm{c}^{2}\right)}\left[\frac{\sqrt{\mathrm{a}^{2}+\mathrm{b}^{2}-\mathrm{c}^{2}}}{\sqrt{3}}\right. \\
& \left.-\sqrt{\mathrm{c}^{2}-\mathrm{b}^{2}-\mathrm{a}^{2}} \tan \left(\frac{\sqrt{\mathrm{c}^{2}-\mathrm{b}^{2}-\mathrm{a}^{2}}}{2}(\xi+\mathrm{C})\right)\right]^{-1}, \\
\mathrm{u}_{13}(\xi)= \pm & \sqrt{-\frac{\alpha}{3 \beta}} \pm \frac{2}{3} \sqrt{\frac{-\alpha}{\beta}\left(\mathrm{a}^{2}-\mathrm{c}^{2}\right)}\left[\frac{\sqrt{\mathrm{a}^{2}-\mathrm{c}^{2}}}{\sqrt{3}}-\sqrt{\mathrm{c}^{2}-\mathrm{a}^{2}} \tan \left(\frac{\sqrt{\mathrm{c}^{2}-\mathrm{a}^{2}}}{2}(\xi+C)\right)\right]^{-1} .
\end{aligned}
$$

By using of the (67) and Family 2, 3 and Family 5 we get

$$
\begin{aligned}
& \mathrm{u}_{14}(\xi)= \pm \sqrt{-\frac{\alpha}{3 \beta}} \pm \frac{2}{3} \sqrt{\frac{-\alpha}{\beta}}\left[\frac{1}{\sqrt{3}}+\tanh \left(\frac{\sqrt{\mathrm{a}^{2}+\mathrm{b}^{2}-\mathrm{c}^{2}}}{2}(\xi+\mathrm{C})\right)\right]^{-1}, \\
& \mathrm{u}_{15}(\xi)= \pm \sqrt{-\frac{\alpha}{3 \beta}} \pm \frac{2}{3} \sqrt{\frac{-\alpha}{\beta}}\left[\frac{1}{\sqrt{3}}+\tanh \left(\frac{\sqrt{\mathrm{a}^{2}+\mathrm{b}^{2}}}{2}(\xi+\mathrm{C})\right)\right]^{-1}, \\
& \mathrm{u}_{16}(\xi)= \pm \sqrt{-\frac{\alpha}{3 \beta}} \pm \frac{2}{3} \sqrt{\frac{-\alpha}{\beta}}\left[\frac{1}{\sqrt{3}}+\tanh \left(\frac{\sqrt{\mathrm{b}^{2}-\mathrm{c}^{2}}}{2}(\xi+\mathrm{C})\right)\right]^{-1}
\end{aligned}
$$

By using of the (61) and Family 6 we get

$$
\mathrm{u}_{17}(\xi)= \pm \sqrt{-\frac{\alpha}{3 \beta}} \pm \frac{2}{3} \sqrt{\frac{-\alpha}{\beta}}\left[\frac{1}{\sqrt{3}}+\tan \left(\frac{1}{2} \arctan \left[\frac{\mathrm{e}^{2 \mathrm{~b}(\xi+\mathrm{C})}-1}{\mathrm{e}^{2 \mathrm{~b}(\xi+\mathrm{C})}+1}, \frac{2 \mathrm{e}^{\mathrm{b}(\xi+\mathrm{C})}}{\mathrm{e}^{2 \mathrm{~b}(\xi+\mathrm{C})}+1}\right]\right)\right]^{-1} .
$$


By using of the (67) and Family 10, 11, 12, 13 and 14 respectively can be written as

$$
\begin{aligned}
& \mathrm{u}_{18}(\xi)= \pm \sqrt{-\frac{\alpha}{3 \beta}} \mp \frac{1}{3} \sqrt{\frac{-\alpha}{\beta}}\left[\frac{1}{2}-\frac{1}{2 \sqrt{3}}-\frac{\mathrm{e}^{\mathrm{ak}(\xi+\mathrm{C})}}{-1+\mathrm{e}^{\mathrm{ak}(\xi+\mathrm{C})}}\right]^{-1}, \\
& \mathrm{u}_{19}(\xi)= \pm \sqrt{-\frac{\alpha}{3 \beta}} \pm \frac{2 \mathrm{~b}}{3} \sqrt{\frac{-\alpha}{\beta}}\left[-\mathrm{a}+\frac{\mathrm{b}}{\sqrt{3}}-\frac{(\mathrm{a}+\mathrm{b}) \mathrm{e}^{\mathrm{b}(\xi+\mathrm{C})}-1}{(\mathrm{a}-\mathrm{b}) \mathrm{e}^{\mathrm{b}(\xi+\mathrm{C})}-1}\right]^{-1}, \\
& \mathrm{u}_{20}(\xi)= \pm \sqrt{-\frac{\alpha}{3 \beta}} \pm \frac{2 \mathrm{~b}}{3} \sqrt{\frac{-\alpha}{\beta}}\left[-\mathrm{c}+\frac{\mathrm{b}}{\sqrt{3}}+\frac{(\mathrm{b}+\mathrm{c}) \mathrm{e}^{\mathrm{b}(\xi+\mathrm{C})}+1}{(\mathrm{~b}-\mathrm{c}) \mathrm{e}^{\mathrm{b}(\xi+\mathrm{C})}-1}\right]^{-1}, \\
& \mathrm{u}_{21}(\xi)= \pm \sqrt{-\frac{\alpha}{3 \beta}} \pm \frac{2 \mathrm{~b}}{3} \sqrt{\frac{-\alpha}{\beta}}\left[-\mathrm{a}+\frac{\mathrm{b}}{\sqrt{3}}+\frac{\mathrm{e}^{\mathrm{b}(\xi+\mathrm{C})}+\mathrm{b}-\mathrm{a}}{\mathrm{e}^{\mathrm{b}(\xi+\mathrm{C})}-\mathrm{b}-\mathrm{a}}\right]^{-1}, \\
& \mathrm{u}_{22}(\xi)= \pm \sqrt{-\frac{\alpha}{3 \beta}} \mp \frac{\mathrm{a}}{3} \sqrt{\frac{-\alpha}{\beta}}\left[\frac{\mathrm{a}}{2}-\frac{\mathrm{a}}{2 \sqrt{3}}-\frac{\mathrm{a} \mathrm{e}^{\mathrm{a}(\xi+\mathrm{C})}}{\mathrm{ce}]^{\mathrm{a}(\xi+\mathrm{C})}-1}\right]^{-1},
\end{aligned}
$$

where $\xi=\mu\left(\mathrm{x}+\mathrm{y} \mp \frac{1}{\mu} \sqrt{\frac{-2 \alpha}{\mathrm{a}^{2}+\mathrm{b}^{2}-\mathrm{c}^{2}}} \mathrm{t}\right)$.

Set III:

$$
\begin{aligned}
\mathrm{m}= & \pm \frac{1}{\mu} \sqrt{\frac{-\alpha}{2\left(\mathrm{a}^{2}+\mathrm{b}^{2}-\mathrm{c}^{2}\right)}}, \quad \mathrm{A}_{1}= \pm \frac{\mathrm{b}-\mathrm{c}}{2} \sqrt{\frac{-\alpha}{\beta\left(\mathrm{a}^{2}+\mathrm{b}^{2}-\mathrm{c}^{2}\right)}} \\
\mathrm{B}_{1}= & \mp \frac{1}{2(\mathrm{~b}-\mathrm{c})} \sqrt{\frac{-\alpha}{\beta\left(\mathrm{a}^{2}+\mathrm{b}^{2}-\mathrm{c}^{2}\right)}}, \\
\mathrm{A}_{0}= & 0, \quad \mu=\mu, \quad \mathrm{p}=-\frac{\mathrm{a}}{\mathrm{b}-\mathrm{c}}, \quad \mathrm{u}(\xi)=\mathrm{A}_{1}\left[\mathrm{p}+\tan \left(\frac{\Phi(\xi)}{2}\right)\right] \\
& +\mathrm{B}_{1}\left[\mathrm{p}+\tan \left(\frac{\Phi(\xi)}{2}\right)\right]^{-1},
\end{aligned}
$$

where a, b and c are arbitrary constants. By using of the (73) and Family 1, 4 respectively get to

$$
\begin{aligned}
\mathrm{u}_{23}(\xi)=\mp & \frac{1}{2} \sqrt{\frac{-\alpha}{\beta\left(\mathrm{a}^{2}+\mathrm{b}^{2}-\mathrm{c}^{2}\right)}}\left[\sqrt{\mathrm{c}^{2}-\mathrm{b}^{2}-\mathrm{a}^{2}} \tan \left(\frac{\sqrt{\mathrm{c}^{2}-\mathrm{b}^{2}-\mathrm{a}^{2}}}{2}(\xi+C)\right)\right. \\
& \left.-\frac{1}{\sqrt{\mathrm{c}^{2}-\mathrm{b}^{2}-\mathrm{a}^{2}}} \cot \left(\frac{\sqrt{\mathrm{c}^{2}-\mathrm{b}^{2}-\mathrm{a}^{2}}}{2}(\xi+C)\right)\right], \\
\mathrm{u}_{24}(\xi)=\mp & \frac{1}{2} \sqrt{\frac{-\alpha}{\beta\left(\mathrm{a}^{2}-\mathrm{c}^{2}\right)}}\left[\sqrt{\mathrm{c}^{2}-\mathrm{a}^{2}} \tan \left(\frac{\sqrt{\mathrm{c}^{2}-\mathrm{a}^{2}}}{2}(\xi+\mathrm{C})\right)\right. \\
& \left.-\frac{1}{\sqrt{\mathrm{c}^{2}-\mathrm{a}^{2}}} \cot \left(\frac{\sqrt{\mathrm{c}^{2}-\mathrm{a}^{2}}}{2}(\xi+\mathrm{C})\right)\right] .
\end{aligned}
$$


By using of the (67) and Family 2, 3 and Family 5 we get

$$
\begin{aligned}
\mathrm{u}_{25}(\xi)= \pm & \frac{1}{2} \sqrt{\frac{-\alpha}{\beta\left(\mathrm{a}^{2}+\mathrm{b}^{2}-\mathrm{c}^{2}\right)}}\left[\sqrt{\mathrm{a}^{2}+\mathrm{b}^{2}-\mathrm{c}^{2}} \tanh \left(\frac{\sqrt{\mathrm{a}^{2}+\mathrm{b}^{2}-\mathrm{c}^{2}}}{2}(\xi+\mathrm{C})\right)\right. \\
& \left.-\frac{1}{\sqrt{\mathrm{a}^{2}+\mathrm{b}^{2}-\mathrm{c}^{2}}} \operatorname{coth}\left(\frac{\sqrt{\mathrm{a}^{2}+\mathrm{b}^{2}-\mathrm{c}^{2}}}{2}(\xi+\mathrm{C})\right)\right], \\
\mathrm{u}_{26}(\xi)= \pm & \frac{1}{2} \sqrt{\frac{-\alpha}{\beta\left(\mathrm{a}^{2}+\mathrm{b}^{2}\right)}}\left[\sqrt{\mathrm{a}^{2}+\mathrm{b}^{2}} \tanh \left(\frac{\sqrt{\mathrm{a}^{2}+\mathrm{b}^{2}}}{2}(\xi+\mathrm{C})\right)\right. \\
& \left.-\frac{1}{\sqrt{\mathrm{a}^{2}+\mathrm{b}^{2}}} \operatorname{coth}\left(\frac{\sqrt{\mathrm{a}^{2}+\mathrm{b}^{2}}}{2}(\xi+\mathrm{C})\right)\right], \\
\mathrm{u}_{27}(\xi)= \pm & \frac{1}{2} \sqrt{\frac{-\alpha}{\beta\left(\mathrm{b}^{2}-\mathrm{c}^{2}\right)}}\left[\sqrt{\mathrm{b}^{2}-\mathrm{c}^{2}} \tanh \left(\frac{\sqrt{\mathrm{b}^{2}-\mathrm{c}^{2}}}{2}(\xi+\mathrm{C})\right)\right. \\
& \left.-\frac{1}{\sqrt{\mathrm{b}^{2}-\mathrm{c}^{2}}} \operatorname{coth}\left(\frac{\sqrt{\mathrm{b}^{2}-\mathrm{c}^{2}}}{2}(\xi+\mathrm{C})\right)\right],
\end{aligned}
$$

By using of the (61) and Family 6 we get

$$
\begin{aligned}
\mathrm{u}_{28}(\xi)= & \pm \sqrt{-\frac{\alpha}{4 \beta}} \tan \left(\frac{1}{2} \arctan \left[\frac{\mathrm{e}^{2 \mathrm{~b}(\xi+\mathrm{C})}-1}{\mathrm{e}^{2 \mathrm{~b}(\xi+\mathrm{C})}+1}, \frac{2 \mathrm{e}^{\mathrm{b}(\xi+\mathrm{C})}}{\mathrm{e}^{2 \mathrm{~b}(\xi+\mathrm{C})}+1}\right]\right) \\
& \mp \frac{1}{\mathrm{~b}^{2}} \sqrt{-\frac{\alpha}{4 \beta}} \cot \left(\frac{1}{2} \arctan \left[\frac{\mathrm{e}^{2 \mathrm{~b}(\xi+\mathrm{C})}-1}{\mathrm{e}^{2 \mathrm{~b}(\xi+\mathrm{C})}+1}, \frac{2 \mathrm{e}^{\mathrm{b}(\xi+\mathrm{C})}}{\mathrm{e}^{2 \mathrm{~b}(\xi+\mathrm{C})}+1}\right]\right) .
\end{aligned}
$$

By using of the (67) and Family 10, 11, 12, 13 and 14 respectively can be written as

$$
\begin{aligned}
& \mathrm{u}_{29}(\xi)=\mp \sqrt{-\frac{\alpha}{\beta}}\left[\frac{1}{2}-\frac{\mathrm{e}^{\mathrm{ak}(\xi+\mathrm{C})}}{-1+\mathrm{e}^{\mathrm{ak}(\xi+\mathrm{C})}}\right] \pm \frac{1}{4 \mathrm{k}^{2} \mathrm{a}^{2}} \sqrt{-\frac{\alpha}{\beta}}\left[\frac{1}{2}-\frac{\mathrm{e}^{\mathrm{ak}(\xi+\mathrm{C})}}{-1+\mathrm{e}^{\mathrm{ak}(\xi+C)}}\right]^{-1}, \\
& \mathrm{u}_{30}(\xi)= \pm \frac{1}{2 \mathrm{~b}} \sqrt{-\frac{\alpha}{\beta}}\left[-\mathrm{a}-\frac{(\mathrm{a}+\mathrm{b}) \mathrm{e}^{\mathrm{b}(\xi+\mathrm{C})}-1}{(\mathrm{a}-\mathrm{b}) \mathrm{e}^{\mathrm{b}(\xi+\mathrm{C})}-1}\right] \mp \frac{1}{2 \mathrm{~b}} \sqrt{-\frac{\alpha}{\beta}}\left[-\mathrm{a}-\frac{(\mathrm{a}+\mathrm{b}) \mathrm{e}^{\mathrm{b}(\xi+\mathrm{C})}-1}{(\mathrm{a}-\mathrm{b}) \mathrm{e}^{\mathrm{b}(\xi+\mathrm{C})}-1}\right]^{-1}, \\
& \mathrm{u}_{31}(\xi)= \pm \frac{1}{2 \mathrm{~b}} \sqrt{-\frac{\alpha}{\beta}}\left[-\mathrm{c}-\frac{(\mathrm{b}+\mathrm{c}) \mathrm{e}^{\mathrm{b}(\xi+\mathrm{C})}+1}{(\mathrm{~b}-\mathrm{c}) \mathrm{e}^{\mathrm{b}(\xi+\mathrm{C})}-1}\right] \mp \frac{1}{2 \mathrm{~b}} \sqrt{-\frac{\alpha}{\beta}}\left[-\mathrm{c}-\frac{(\mathrm{b}+\mathrm{c}) \mathrm{e}^{\mathrm{b}(\xi+\mathrm{C})}+1}{(\mathrm{~b}-\mathrm{c}) \mathrm{e}^{\mathrm{b}(\xi+\mathrm{C})}-1}\right]^{-1}, \\
& \mathrm{u}_{32}(\xi)= \pm \frac{1}{2 \mathrm{~b}} \sqrt{-\frac{\alpha}{\beta}}\left[-\mathrm{a}-\frac{\mathrm{e}^{\mathrm{b}(\xi+\mathrm{C})}+\mathrm{b}-\mathrm{a}}{\left(\mathrm{e}^{\mathrm{b}(\xi+\mathrm{C})}-\mathrm{b}-\mathrm{a}\right.}\right] \mp \frac{1}{2 \mathrm{~b}} \sqrt{-\frac{\alpha}{\beta}}\left[-\mathrm{a}-\frac{\mathrm{e}^{\mathrm{b}(\xi+\mathrm{C})}+\mathrm{b}-\mathrm{a}}{\mathrm{e}^{\mathrm{b}(\xi+\mathrm{C})}-\mathrm{b}-\mathrm{a}}\right]^{-1}, \\
& \mathrm{u}_{33}(\xi)=\mp \frac{1}{\mathrm{a}} \sqrt{-\frac{\alpha}{\beta}}\left[\frac{\mathrm{a}}{2}-\frac{\mathrm{a} \mathrm{e}^{\mathrm{a}(\xi+\mathrm{C})}}{\mathrm{ce}^{\mathrm{a}(\xi+\mathrm{C})}-1}\right] \pm \frac{1}{2 \mathrm{a}} \sqrt{-\frac{\alpha}{\beta}}\left[\frac{\mathrm{a}}{2}-\frac{\mathrm{ae}^{\mathrm{a}(\xi+\mathrm{C})}}{\mathrm{ce}^{\mathrm{a}(\xi+\mathrm{C})}-1}\right]^{-1},
\end{aligned}
$$

where $\xi=\mu\left(\mathrm{x}+\mathrm{y} \mp \frac{1}{\mu} \sqrt{\frac{-\alpha}{2\left(\mathrm{a}^{2}+\mathrm{b}^{2}-\mathrm{c}^{2}\right)}} \mathrm{t}\right)$. 
Set IV:

$$
\begin{aligned}
& \mathrm{m}= \pm \frac{1}{\mu} \sqrt{\frac{\alpha}{\mathrm{a}^{2}+\mathrm{b}^{2}-\mathrm{c}^{2}}}, \quad \mathrm{~A}_{1}= \pm(\mathrm{b}-\mathrm{c}) \sqrt{\frac{\alpha}{2 \beta\left(\mathrm{a}^{2}+\mathrm{b}^{2}-\mathrm{c}^{2}\right)}}, \\
& \mathrm{B}_{1}=\mp \frac{1}{2(\mathrm{~b}-\mathrm{c})} \sqrt{\frac{2 \alpha\left(\mathrm{a}^{2}+\mathrm{b}^{2}-\mathrm{c}^{2}\right)}{\beta}}, \\
& \mathrm{A}_{0}=0, \quad \mu=\mu, \quad \mathrm{p}=-\frac{\mathrm{a}}{\mathrm{b}-\mathrm{c}}, \\
& \mathrm{u}(\xi)=A_{1}\left[p+\tan \left(\frac{\Phi(\xi)}{2}\right)\right]+\mathrm{B}_{1}\left[\mathrm{p}+\tan \left(\frac{\Phi(\xi)}{2}\right)\right]^{-1},
\end{aligned}
$$

where a, b and c are arbitrary constants. By using of the (79) and Family $\mathbf{1 ,} \mathbf{4}$ respectively get to

$$
\begin{aligned}
& \mathrm{u}_{33}(\xi)=\mp \sqrt{\frac{-\alpha}{2 \beta}}\left[\tan \left(\frac{\sqrt{\mathrm{c}^{2}-\mathrm{b}^{2}-\mathrm{a}^{2}}}{2}(\xi+\mathrm{C})\right)-\cot \left(\frac{\sqrt{\mathrm{c}^{2}-\mathrm{b}^{2}-\mathrm{a}^{2}}}{2}(\xi+\mathrm{C})\right)\right], \\
& \mathrm{u}_{34}(\xi)=\mp \sqrt{\frac{-\alpha}{2 \beta}}\left[\tan \left(\frac{\sqrt{\mathrm{c}^{2}-\mathrm{a}^{2}}}{2}(\xi+\mathrm{C})\right)-\cot \left(\frac{\sqrt{\mathrm{c}^{2}-\mathrm{a}^{2}}}{2}(\xi+\mathrm{C})\right)\right] .
\end{aligned}
$$

By using of the (79) and Family 2, 3 and Family 5 we get

$$
\begin{aligned}
& \mathrm{u}_{35}(\xi)= \pm \sqrt{\frac{-\alpha}{2 \beta}}\left[\tanh \left(\frac{\sqrt{\mathrm{a}^{2}+\mathrm{b}^{2}-\mathrm{c}^{2}}}{2}(\xi+\mathrm{C})\right)-\operatorname{coth}\left(\frac{\sqrt{\mathrm{a}^{2}+\mathrm{b}^{2}-\mathrm{c}^{2}}}{2}(\xi+\mathrm{C})\right)\right], \\
& \mathrm{u}_{36}(\xi)= \pm \sqrt{\frac{-\alpha}{2 \beta}}\left[\tanh \left(\frac{\sqrt{\mathrm{a}^{2}+\mathrm{b}^{2}}}{2}(\xi+\mathrm{C})\right)-\operatorname{coth}\left(\frac{\sqrt{\mathrm{a}^{2}+\mathrm{b}^{2}}}{2}(\xi+\mathrm{C})\right)\right], \\
& \mathrm{u}_{37}(\xi)= \pm \sqrt{\frac{-\alpha}{2 \beta}}\left[\tanh \left(\frac{\sqrt{\mathrm{b}^{2}-\mathrm{c}^{2}}}{2}(\xi+\mathrm{C})\right)-\operatorname{coth}\left(\frac{\sqrt{\mathrm{b}^{2}-\mathrm{c}^{2}}}{2}(\xi+\mathrm{C})\right)\right] .
\end{aligned}
$$

By using of the (79) and Family 6 we get

$$
\begin{aligned}
& \mathrm{u}_{38}(\xi)= \pm \sqrt{-\frac{\alpha}{2 \beta}} \tan \left(\frac{1}{2} \arctan \left[\frac{\mathrm{e}^{2 \mathrm{~b}(\xi+\mathrm{C})}-1}{\mathrm{e}^{2 \mathrm{~b}(\xi+\mathrm{C})}+1}, \frac{2 \mathrm{e}^{\mathrm{b}(\xi+\mathrm{C})}}{\mathrm{e}^{2 \mathrm{~b}(\xi+\mathrm{C})}+1}\right]\right) \\
& \mp \sqrt{\frac{\alpha}{2 \beta}} \cot \left(\frac{1}{2} \arctan \left[\frac{\mathrm{e}^{2 \mathrm{~b}(\xi+\mathrm{C})}-1}{\mathrm{e}^{2 \mathrm{~b}(\xi+\mathrm{C})}+1}, \frac{2 \mathrm{e}^{\mathrm{b}(\xi+\mathrm{C})}}{\mathrm{e}^{2 \mathrm{~b}(\xi+\mathrm{C})}+1}\right]\right) .
\end{aligned}
$$

By using of the (79) and Family 10, 11, 12, 13 and 14 respectively can be written as

$$
\begin{aligned}
& \mathrm{u}_{39}(\xi)=\mp \sqrt{\frac{2 \alpha}{\beta}}\left[\frac{1}{2}-\frac{\mathrm{e}^{\mathrm{ak}(\xi+\mathrm{C})}}{-1+\mathrm{e}^{\mathrm{ak}(\xi+\mathrm{C})}}\right] \pm \frac{1}{4} \sqrt{\frac{2 \alpha}{\beta}}\left[\frac{1}{2}-\frac{\mathrm{e}^{\mathrm{ak}(\xi+\mathrm{C})}}{-1+\mathrm{e}^{\mathrm{ak}(\xi+\mathrm{C})}}\right]^{-1}, \\
& \mathrm{u}_{40}(\xi)= \pm \frac{1}{\mathrm{~b}} \sqrt{\frac{\alpha}{2 \beta}}\left[-\mathrm{a}-\frac{(\mathrm{a}+\mathrm{b}) \mathrm{e}^{\mathrm{b}(\xi+\mathrm{C})}-1}{(\mathrm{a}-\mathrm{b}) \mathrm{e}^{\mathrm{b}(\xi+\mathrm{C})}-1}\right] \mp \mathrm{b} \sqrt{\frac{\alpha}{2 \beta}}\left[-\mathrm{a}-\frac{(\mathrm{a}+\mathrm{b}) \mathrm{e}^{\mathrm{b}(\xi+\mathrm{C})}-1}{(\mathrm{a}-\mathrm{b}) \mathrm{e}^{\mathrm{b}(\xi+\mathrm{C})}-1}\right]^{-1}, \\
& \mathrm{u}_{41}(\xi)= \pm \frac{1}{\mathrm{~b}} \sqrt{\frac{\alpha}{2 \beta}}\left[-\mathrm{c}-\frac{(\mathrm{b}+\mathrm{c}) \mathrm{e}^{\mathrm{b}(\xi+\mathrm{C})}+1}{(\mathrm{~b}-\mathrm{c}) \mathrm{e}^{\mathrm{b}(\xi+\mathrm{C})}-1}\right] \mp \mathrm{b} \sqrt{\frac{\alpha}{2 \beta}}\left[-\mathrm{c}-\frac{(\mathrm{b}+\mathrm{c}) \mathrm{e}^{\mathrm{b}(\xi+\mathrm{C})}+1}{(\mathrm{~b}-\mathrm{c}) \mathrm{e}^{\mathrm{b}(\xi+\mathrm{C})}-1}\right]^{-1},
\end{aligned}
$$




$$
\begin{aligned}
& \mathrm{u}_{42}(\xi)= \pm \frac{1}{\mathrm{~b}} \sqrt{\frac{\alpha}{2 \beta}}\left[-\mathrm{a}-\frac{\mathrm{e}^{\mathrm{b}(\xi+\mathrm{C})}+\mathrm{b}-\mathrm{a}}{\left(\mathrm{e}^{\mathrm{b}(\xi+\mathrm{C})}-\mathrm{b}-\mathrm{a}\right.}\right] \mp \frac{1}{\mathrm{~b}} \sqrt{\frac{\alpha}{2 \beta}}\left[-\mathrm{a}-\frac{\mathrm{e}^{\mathrm{b}(\xi+\mathrm{C})}+\mathrm{b}-\mathrm{a}}{\mathrm{e}^{\mathrm{b}(\xi+\mathrm{C})}-\mathrm{b}-\mathrm{a}}\right]^{-1}, \\
& \mathrm{u}_{43}(\xi)=\mp \sqrt{\frac{\alpha}{2 \beta}}\left[1-\frac{\mathrm{ae}^{\mathrm{a}(\xi+\mathrm{C})}}{\mathrm{ce}^{\mathrm{a}(\xi+\mathrm{C})}-1}\right] \pm \sqrt{\frac{\alpha}{2 \beta}}\left[1-\frac{\mathrm{ae}^{\mathrm{a}(\xi+\mathrm{C})}}{\mathrm{ce}^{\mathrm{a}(\xi+\mathrm{C})}-1}\right]^{-1},
\end{aligned}
$$

where $\xi=\mu\left(\mathrm{x}+\mathrm{y} \mp \frac{1}{\mu} \sqrt{\frac{\alpha}{\mathrm{a}^{2}+\mathrm{b}^{2}-\mathrm{c}^{2}}} \mathrm{t}\right)$.

Set V:

$$
\begin{aligned}
& \mathrm{m}= \pm \frac{1}{\mu} \sqrt{\frac{-2 \alpha}{\mathrm{a}^{2}+\mathrm{b}^{2}-\mathrm{c}^{2}}}, \quad \mathrm{~A}_{0}= \pm(\mathrm{a}+\mathrm{p}(\mathrm{b}-\mathrm{c})) \sqrt{-\frac{\alpha}{\beta\left(\mathrm{a}^{2}+\mathrm{b}^{2}-\mathrm{c}^{2}\right)}} \\
& \mathrm{A}_{1}= \pm(\mathrm{b}-\mathrm{c}) \sqrt{-\frac{\alpha}{\beta\left(\mathrm{a}^{2}+\mathrm{b}^{2}-\mathrm{c}^{2}\right)}} \\
& \mathrm{B}_{1}=0, \quad \mu=\mu, \quad \mathrm{p}=\mathrm{p}, \quad \mathrm{u}(\xi)=\mathrm{A}_{0}+\mathrm{A}_{1}\left[\mathrm{p}+\tan \left(\frac{\Phi(\xi)}{2}\right)\right],
\end{aligned}
$$

where a, b and c are arbitrary constants. By using of the (85) and Family $\mathbf{1}, \mathbf{4}$ respectively get to

$$
\begin{aligned}
u_{44}(\xi)= & \pm \sqrt{-\frac{\alpha}{\beta\left(a^{2}+b^{2}-c^{2}\right)}}[2 a+2 p(b-c) \\
& \left.-\sqrt{c^{2}-b^{2}-a^{2}} \tan \left(\frac{\sqrt{c^{2}-b^{2}-a^{2}}}{2}(\xi+C)\right)\right], \\
\mathrm{u}_{45}(\xi) & = \pm \sqrt{-\frac{\alpha}{\beta\left(\mathrm{a}^{2}-\mathrm{c}^{2}\right)}}\left[2 \mathrm{a}-2 \mathrm{pc}-\sqrt{\mathrm{c}^{2}-\mathrm{a}^{2}} \tan \left(\frac{\sqrt{\mathrm{c}^{2}-\mathrm{a}^{2}}}{2}(\xi+\mathrm{C})\right)\right]
\end{aligned}
$$

By using of the (67) and Family 2, 3 and Family 5 we get

$$
\begin{aligned}
& u_{46}(\xi)= \pm \sqrt{-\frac{\alpha}{\beta\left(a^{2}+b^{2}-c^{2}\right)}}[2 a+2 p(b-c) \\
&\left.+\sqrt{a^{2}+b^{2}-c^{2}} \tanh \left(\frac{\sqrt{a^{2}+b^{2}-c^{2}}}{2}(\xi+C)\right)\right] \\
& \mathrm{u}_{47}(\xi)= \pm \sqrt{-\frac{\alpha}{\beta\left(\mathrm{a}^{2}+\mathrm{b}^{2}\right)}}\left[2 \mathrm{a}+2 \mathrm{pb}+\sqrt{\mathrm{a}^{2}+\mathrm{b}^{2}} \tanh \left(\frac{\sqrt{\mathrm{a}^{2}+\mathrm{b}^{2}}}{2}(\xi+\mathrm{C})\right)\right] \\
& \mathrm{u}_{48}(\xi)= \pm \sqrt{-\frac{\alpha}{\beta\left(\mathrm{b}^{2}-\mathrm{c}^{2}\right)}}\left[2 \mathrm{p}(\mathrm{b}-\mathrm{c})+\sqrt{\mathrm{b}^{2}-\mathrm{c}^{2}} \tanh \left(\frac{\sqrt{\mathrm{b}^{2}-\mathrm{c}^{2}}}{2}(\xi+\mathrm{C})\right)\right] .
\end{aligned}
$$

By using of the (61) and Family 6 we get

$$
\mathrm{u}_{49}(\xi)= \pm \sqrt{-\frac{\alpha}{\beta}}\left[2 \mathrm{p}+\tan \left(\frac{1}{2} \arctan \left[\frac{\mathrm{e}^{2 \mathrm{~b}(\xi+\mathrm{C})}-1}{\mathrm{e}^{2 \mathrm{~b}(\xi+\mathrm{C})}+1}, \frac{2 \mathrm{e}^{\mathrm{b}(\xi+\mathrm{C})}}{\mathrm{e}^{2 \mathrm{~b}(\xi+\mathrm{C})}+1}\right]\right)\right]
$$


By using of the (67) and Family 10, 11, 12, 13 and 14 respectively can be written as

$$
\begin{aligned}
& \mathrm{u}_{50}(\xi)= \pm \sqrt{-\frac{\alpha}{\beta}}\left[1-4 \mathrm{p}+2 \frac{\mathrm{e}^{\mathrm{ak}(\xi+\mathrm{C})}}{-1+\mathrm{e}^{\mathrm{ak}(\xi+\mathrm{C})}}\right], \\
& \mathrm{u}_{51}(\xi)= \pm \frac{1}{\mathrm{~b}} \sqrt{-\frac{\alpha}{\beta}}\left[\mathrm{a}+2 \mathrm{p}(\mathrm{b}-\mathrm{a})-(\mathrm{b}-\mathrm{a}) \frac{(\mathrm{a}+\mathrm{b}) \mathrm{e}^{\mathrm{b}(\xi+C)}-1}{(\mathrm{a}-\mathrm{b}) \mathrm{e}^{\mathrm{b}(\xi+C)}-1}\right], \\
& \mathrm{u}_{52}(\xi)= \pm \frac{1}{\mathrm{~b}} \sqrt{-\frac{\alpha}{3 \beta}}\left[\mathrm{c}+2 \mathrm{p}(\mathrm{b}-\mathrm{c})+(\mathrm{b}-\mathrm{c}) \frac{(\mathrm{b}+\mathrm{c}) \mathrm{e}^{\mathrm{b}(\xi+C)}+1}{(\mathrm{~b}-\mathrm{c}) \mathrm{e}^{\mathrm{b}(\xi+C)}-1}\right], \\
& \mathrm{u}_{53}(\xi)= \pm \frac{1}{\mathrm{~b}} \sqrt{-\frac{\alpha}{\beta}}\left[\mathrm{a}+2 \mathrm{p}(\mathrm{b}+\mathrm{a})+(\mathrm{b}+\mathrm{a}) \frac{\mathrm{e}^{\mathrm{b}(\xi+C)}+\mathrm{b}-\mathrm{a}}{\mathrm{e}^{\mathrm{b}(\xi+C)}-\mathrm{b}-\mathrm{a}}\right], \\
& \mathrm{u}_{54}(\xi)= \pm \frac{1}{\mathrm{a}} \sqrt{-\frac{\alpha}{\beta}}\left[\mathrm{a}-4 \mathrm{pc}+2 \mathrm{c} \frac{\mathrm{a} \mathrm{e}^{\mathrm{a}(\xi+\mathrm{C})}}{\mathrm{ce}}\right],
\end{aligned}
$$

where $\xi=\mu\left(\mathrm{x}+\mathrm{y} \mp \frac{1}{\mu} \sqrt{\frac{-2 \alpha}{\mathrm{a}^{2}+\mathrm{b}^{2}-\mathrm{c}^{2}}} \mathrm{t}\right)$.

\section{The SRLW Equation}

We consider last example the nonlinear SRLW equation as follows

$$
\mathrm{u}_{\mathrm{tt}}+\mathrm{u}_{\mathrm{xx}}+\mathrm{u}_{\mathrm{xxtt}}+\left(\mathrm{uu}_{\mathrm{x}}\right)_{\mathrm{t}}=0,
$$

by using the wave variable $\xi=\mu(\mathrm{x}-\mathrm{mt})$ reduce it to an ODE as follows

$$
\left(\mathrm{m}^{2}+1\right) \mathrm{u}+\mu^{2} \mathrm{~m}^{2} \mathrm{u}^{\prime \prime}-\frac{\mathrm{m}}{2} \mathrm{u}^{2}=0,
$$

where obtained by twice integrating and neglecting the constant of integration. Balancing the $\mathrm{u}^{\prime \prime}$ and $\mathrm{u}^{2}$ by using homogenous principal, we have

$$
\mathrm{M}+2=2 \mathrm{M}, \quad \Rightarrow \mathrm{M}=2 .
$$

Then the trail solution is

$$
\begin{aligned}
\mathrm{u}(\xi)=\mathrm{A}_{0} & +\mathrm{A}_{1}\left[\mathrm{p}+\tan \left(\frac{\Phi(\xi)}{2}\right)\right]+\mathrm{A}_{2}\left[\mathrm{p}+\tan \left(\frac{\Phi(\xi)}{2}\right)\right]^{2} \\
& +\mathrm{B}_{1}\left[\mathrm{p}+\tan \left(\frac{\Phi(\xi)}{2}\right)\right]^{-1}+\mathrm{B}_{2}\left[\mathrm{p}+\tan \left(\frac{\Phi(\xi)}{2}\right)\right]^{-2} .
\end{aligned}
$$

For simplicity we set $\mathrm{p}=1$, then Eq. (93) is simplified as follows

$$
\begin{aligned}
\mathrm{u}(\xi)=\mathrm{A}_{0} & +\mathrm{A}_{1}\left[1+\tan \left(\frac{\Phi(\xi)}{2}\right)\right]+\mathrm{A}_{2}\left[1+\tan \left(\frac{\Phi(\xi)}{2}\right)\right]^{2} \\
& +\mathrm{B}_{1}\left[1+\tan \left(\frac{\Phi(\xi)}{2}\right)\right]^{-1}+\mathrm{B}_{2}\left[1+\tan \left(\frac{\Phi(\xi)}{2}\right)\right]^{-2} .
\end{aligned}
$$

Substituting (94) and (7) into Eq. (91) and by using the well-known Maple software, we obtain the following sets of non-trivial solutions 
Set I:

$$
\begin{gathered}
m=m, \quad \mu= \pm \frac{1}{m} \sqrt{\frac{-\left(m^{2}+1\right)}{a^{2}+b^{2}-c^{2}}} \\
A_{0}=\frac{\left(m^{2}+1\right)\left(2 b^{2}-6 b c+6 a b+2 a^{2}+4 c^{2}-6 a c\right)}{m\left(a^{2}+b^{2}-c^{2}\right)}, \\
B_{1}=0, \quad B_{2}=0, \quad A_{1}=\frac{-6(b-c)(a+b-c)\left(m^{2}+1\right)}{m\left(a^{2}+b^{2}-c^{2}\right)}, \\
A_{2}=\frac{3(b-c)^{2}\left(m^{2}+1\right)}{m\left(a^{2}+b^{2}-c^{2}\right)}, \\
\mathrm{u}(\xi)=\mathrm{A}_{0}+\mathrm{A}_{1}\left[1+\tan \left(\frac{\Phi(\xi)}{2}\right)\right]+\mathrm{A}_{2}\left[1+\tan \left(\frac{\Phi(\xi)}{2}\right)\right]^{2},
\end{gathered}
$$

where a, b and c are arbitrary constants. By using of the (96) and Family 1, 4 respectively get to

$$
\begin{aligned}
\mathrm{u}_{1}(\xi)= & \frac{\left(\mathrm{m}^{2}+1\right)}{\mathrm{m}\left(\mathrm{a}^{2}+\mathrm{b}^{2}-\mathrm{c}^{2}\right)}\left\{2 \mathrm{a}^{2}-4 \mathrm{~b}^{2}+6 \mathrm{bc}-2 \mathrm{c}^{2}-6(\mathrm{a}+\mathrm{b}-\mathrm{c})\right. \\
& \times\left[\mathrm{a}-\sqrt{\mathrm{c}^{2}-\mathrm{b}^{2}-\mathrm{a}^{2}} \tan \left(\frac{\sqrt{\mathrm{c}^{2}-\mathrm{b}^{2}-\mathrm{a}^{2}}}{2}(\xi+C)\right)\right] \\
& \left.+3\left[\mathrm{a}+\mathrm{b}-\mathrm{c}-\sqrt{\mathrm{c}^{2}-\mathrm{b}^{2}-\mathrm{a}^{2}} \tan \left(\frac{\sqrt{\mathrm{c}^{2}-\mathrm{b}^{2}-\mathrm{a}^{2}}}{2}(\xi+C)\right)\right]^{2}\right\} \\
\mathrm{u}_{2}(\xi)= & \frac{\left(\mathrm{m}^{2}+1\right)}{\mathrm{m}\left(\mathrm{a}^{2}-\mathrm{c}^{2}\right)}\left\{2 \mathrm{a}^{2}-\mathrm{c}^{2}-6(\mathrm{a}-\mathrm{c})\left[\mathrm{a}-\sqrt{\mathrm{c}^{2}-\mathrm{a}^{2}} \tan \left(\frac{\sqrt{\mathrm{c}^{2}-\mathrm{a}^{2}}}{2}(\xi+C)\right)\right]\right. \\
& \left.+3\left[\mathrm{a}-\mathrm{c}-\sqrt{\mathrm{c}^{2}-\mathrm{a}^{2}} \tan \left(\frac{\sqrt{\mathrm{c}^{2}-\mathrm{a}^{2}}}{2}(\xi+C)\right)\right]^{2}\right\}
\end{aligned}
$$

By using of the (96) and Family 2, 3 and $\mathbf{5}$ respectively get to

$$
\begin{aligned}
u_{3}(\xi)= & \frac{\left(m^{2}+1\right)}{m\left(a^{2}+b^{2}-c^{2}\right)}\left\{2 a^{2}-4 b^{2}+6 b c-2 c^{2}-6(a+b-c)\right. \\
& \times\left[a+\sqrt{a^{2}+b^{2}-c^{2}} \tanh \left(\frac{\sqrt{a^{2}+b^{2}-c^{2}}}{2}(\xi+C)\right)\right] \\
& \left.+3\left[a+b-c+\sqrt{a^{2}+b^{2}-c^{2}} \tanh \left(\frac{\sqrt{a^{2}+b^{2}-c^{2}}}{2}(\xi+C)\right)\right]^{2}\right\}, \\
u_{4}(\xi)= & \frac{\left(m^{2}+1\right)}{m\left(a^{2}+b^{2}\right)}\left\{2 a^{2}-4 b^{2}-6(a+b)\left[a+\sqrt{a^{2}+b^{2}} \tanh \left(\frac{\sqrt{a^{2}+b^{2}}}{2}(\xi+C)\right)\right]\right. \\
& \left.+3\left[a+b+\sqrt{a^{2}+b^{2}} \tanh \left(\frac{\sqrt{a^{2}+b^{2}}}{2}(\xi+C)\right)\right]^{2}\right\},
\end{aligned}
$$




$$
\begin{gathered}
\mathrm{u}_{5}(\xi)=\frac{\left(\mathrm{m}^{2}+1\right)}{\mathrm{m}\left(\mathrm{b}^{2}-\mathrm{c}^{2}\right)}\left\{-4 \mathrm{~b}^{2}+6 \mathrm{bc}-2 \mathrm{c}^{2}-6(\mathrm{~b}-\mathrm{c})\left[\sqrt{\mathrm{b}^{2}-\mathrm{c}^{2}} \tanh \left(\frac{\sqrt{\mathrm{b}^{2}-\mathrm{c}^{2}}}{2}(\xi+C)\right)\right]\right. \\
\left.+3\left[\mathrm{~b}-\mathrm{c}+\sqrt{\mathrm{b}^{2}-\mathrm{c}^{2}} \tanh \left(\frac{\sqrt{\mathrm{b}^{2}-\mathrm{c}^{2}}}{2}(\xi+C)\right)\right]^{2}\right\} .
\end{gathered}
$$

By using of the (96) and Family 6 we get

$$
\begin{gathered}
\mathrm{u}_{6}(\xi)=\frac{\left(\mathrm{m}^{2}+1\right)}{\mathrm{m}}\left\{-4-6 \tan \left(\frac{1}{2} \arctan \left[\frac{\mathrm{e}^{2 \mathrm{~b}(\xi+\mathrm{C})}-1}{\mathrm{e}^{2 \mathrm{~b}(\xi+\mathrm{C})}+1}, \frac{2 \mathrm{e}^{\mathrm{b}(\xi+\mathrm{C})}}{\mathrm{e}^{2 \mathrm{~b}(\xi+\mathrm{C})}+1}\right]\right)\right. \\
\left.+3\left[1+\tan \left(\frac{1}{2} \arctan \left[\frac{\mathrm{e}^{2 \mathrm{~b}(\xi+\mathrm{C})}-1}{\mathrm{e}^{2 \mathrm{~b}(\xi+\mathrm{C})}+1}, \frac{2 \mathrm{e}^{\mathrm{b}(\xi+\mathrm{C})}}{\mathrm{e}^{2 \mathrm{~b}(\xi+\mathrm{C})}+1}\right]\right)\right]^{2}\right\} .
\end{gathered}
$$

By using of the (96) and Family 10, 11, 12, 13 and 14 respectively can be written as

$$
\begin{aligned}
& \mathrm{u}_{7}(\xi)=\frac{\left(\mathrm{m}^{2}+1\right)}{\mathrm{m}}\left\{-10+12 \frac{\mathrm{e}^{\mathrm{ka}(\xi+\mathrm{C})}}{\left[\mathrm{e}^{\mathrm{ka}(\xi+\mathrm{C})}-1\right]}+12\left[1-\frac{\mathrm{e}^{\mathrm{ka}(\xi+\mathrm{C})}}{\left[\mathrm{e}^{\mathrm{ka}(\xi+\mathrm{C})}-1\right]}\right]^{2}\right\}, \\
& \mathrm{u}_{8}(\xi)=\frac{\left(\mathrm{m}^{2}+1\right)}{\mathrm{m}}\left\{\frac{6 \mathrm{ab}-2 \mathrm{a}^{2}-4 \mathrm{~b}^{2}}{\mathrm{~b}^{2}}-\frac{6(\mathrm{~b}-\mathrm{a})}{\mathrm{b}} \frac{(\mathrm{a}+\mathrm{b}) \mathrm{e}^{\mathrm{b}(\xi+\mathrm{C})}-1}{(\mathrm{a}-\mathrm{b}) \mathrm{e}^{\mathrm{b}(\xi+\mathrm{C})}-1}\right. \\
& \left.+\frac{3(\mathrm{~b}-\mathrm{a})^{2}}{\mathrm{~b}^{2}}\left[1-\frac{(\mathrm{a}+\mathrm{b}) \mathrm{e}^{\mathrm{b}(\xi+\mathrm{C})}-1}{(\mathrm{a}-\mathrm{b}) \mathrm{e}^{\mathrm{b}(\xi+\mathrm{C})}-1}\right]^{2}\right\} \\
& \mathrm{u}_{9}(\xi)=\frac{\left(\mathrm{m}^{2}+1\right)}{\mathrm{m}}\left\{\frac{6 \mathrm{c}-4 \mathrm{~b}}{\mathrm{~b}}-\frac{6(\mathrm{~b}-\mathrm{c})}{\mathrm{b}} \frac{(\mathrm{b}+\mathrm{c}) \mathrm{e}^{\mathrm{b}(\xi+\mathrm{C})}+1}{(\mathrm{~b}-\mathrm{c}) \mathrm{e}^{\mathrm{b}(\xi+\mathrm{C})}-1}\right. \\
& \left.+\frac{3(\mathrm{~b}-\mathrm{c})^{2}}{\mathrm{~b}^{2}}\left[1+\frac{(\mathrm{b}+\mathrm{c}) \mathrm{e}^{\mathrm{b}(\xi+\mathrm{C})}+1}{(\mathrm{~b}-\mathrm{c}) \mathrm{e}^{\mathrm{b}(\xi+\mathrm{C})}-1}\right]^{2}\right\} \\
& \mathrm{u}_{10}(\xi)=\frac{\left(\mathrm{m}^{2}+1\right)}{\mathrm{m}}\left\{-\frac{4 \mathrm{~b}+6 \mathrm{a}}{\mathrm{b}}-\frac{6(\mathrm{~b}+\mathrm{a})(\mathrm{b}+2 \mathrm{a})}{\mathrm{b}^{2}} \frac{\mathrm{e}^{\mathrm{b}(\xi+\mathrm{C})}+\mathrm{b}-\mathrm{a}}{\mathrm{e}^{\mathrm{b}(\xi+C)}-\mathrm{b}-\mathrm{a}}\right. \\
& \left.+\frac{3(\mathrm{~b}+\mathrm{a})^{2}}{\mathrm{~b}^{2}}\left[1+\frac{\mathrm{e}^{\mathrm{b}(\xi+\mathrm{C})}+\mathrm{b}-\mathrm{a}}{\mathrm{e}^{\mathrm{b}(\xi+\mathrm{C})}-\mathrm{b}-\mathrm{a}}\right]^{2}\right\} \\
& \mathrm{u}_{11}(\xi)=\frac{\left(\mathrm{m}^{2}+1\right)}{\mathrm{m}}\left\{\frac{2 \mathrm{a}^{2}-12 \mathrm{c}^{2}}{\mathrm{a}^{2}}-\frac{12 \mathrm{c}(\mathrm{a}-2 \mathrm{c})}{\mathrm{a}^{2}} \frac{\mathrm{a}}{\mathrm{ce}^{\mathrm{a}(\xi+\mathrm{C})}-1}\right.
\end{aligned}
$$

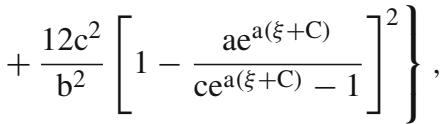

where $\xi=\frac{1}{m} \sqrt{\frac{-\left(m^{2}+1\right)}{a^{2}+b^{2}-c^{2}}}(x-m t)$. 


\section{Set II:}

$$
\begin{gathered}
\mathrm{m}=\mathrm{m}, \quad \mu= \pm \frac{1}{\mathrm{~m}} \sqrt{-\frac{\mathrm{m}^{2}+1}{\mathrm{a}^{2}+\mathrm{b}^{2}-\mathrm{c}^{2}}}, \quad \mathrm{~A}_{0}=\frac{-6\left(\mathrm{~m}^{2}+1\right)(\mathrm{a}-\mathrm{c})(\mathrm{b}-\mathrm{c})}{\mathrm{m}\left(\mathrm{a}^{2}+\mathrm{b}^{2}-\mathrm{c}^{2}\right)}, \quad \\
\mathrm{B}_{1}=\frac{12(\mathrm{a}-\mathrm{c})(\mathrm{a}+\mathrm{b}-\mathrm{c})\left(\mathrm{m}^{2}+1\right)}{\mathrm{m}\left(\mathrm{a}^{2}+\mathrm{b}^{2}-\mathrm{c}^{2}\right)}, \quad \mathrm{B}_{2}=\frac{-12(\mathrm{a}-\mathrm{c})^{2}\left(\mathrm{~m}^{2}+1\right)}{\mathrm{m}\left(\mathrm{a}^{2}+\mathrm{b}^{2}-\mathrm{c}^{2}\right)}, \quad \mathrm{A}_{1}=0, \quad \mathrm{~A}_{2}=0, \\
\mathrm{u}(\xi)=\mathrm{A}_{0}+\mathrm{B}_{1}\left[1+\tan \left(\frac{\Phi(\xi)}{2}\right)\right]^{-1}+\mathrm{B}_{2}\left[1+\tan \left(\frac{\Phi(\xi)}{2}\right)\right]^{-2},
\end{gathered}
$$

where a, b and c are arbitrary constants. By using of the (102) and Family $\mathbf{1 ,} \mathbf{4}$ respectively get to

$$
\begin{aligned}
\mathrm{u}_{12}(\xi)= & \frac{\left(\mathrm{m}^{2}+1\right)}{\mathrm{m}\left(\mathrm{a}^{2}+\mathrm{b}^{2}-\mathrm{c}^{2}\right)}\left\{-6(\mathrm{a}-\mathrm{c})(\mathrm{b}-\mathrm{c})+12(\mathrm{a}-\mathrm{c})(\mathrm{a}+\mathrm{b}-\mathrm{c})\left[1+\frac{\mathrm{a}}{\mathrm{b}-\mathrm{c}}\right.\right. \\
& \left.-\frac{\sqrt{\mathrm{c}^{2}-\mathrm{b}^{2}-\mathrm{a}^{2}}}{\mathrm{~b}-\mathrm{c}} \tan \left(\frac{\sqrt{\mathrm{c}^{2}-\mathrm{b}^{2}-\mathrm{a}^{2}}}{2}(\xi+\mathrm{C})\right)\right]^{-1}-12(\mathrm{a}-\mathrm{c})^{2}\left[1+\frac{\mathrm{a}}{\mathrm{b}-\mathrm{c}}\right. \\
& \left.\left.-\frac{\sqrt{\mathrm{c}^{2}-\mathrm{b}^{2}-\mathrm{a}^{2}}}{\mathrm{~b}-\mathrm{c}} \tan \left(\frac{\sqrt{\mathrm{c}^{2}-\mathrm{b}^{2}-\mathrm{a}^{2}}}{2}(\xi+\mathrm{C})\right)\right]^{-2}\right\}, \\
\mathrm{u}_{13}(\xi)= & \frac{\left(\mathrm{m}^{2}+1\right)}{\mathrm{m}\left(\mathrm{a}^{2}-\mathrm{c}^{2}\right)}\left\{6(\mathrm{a}-\mathrm{c}) \mathrm{c}+12(\mathrm{a}-\mathrm{c})(\mathrm{a}-\mathrm{c})\left[1-\frac{\mathrm{a}}{\mathrm{c}}+\frac{\sqrt{\mathrm{c}^{2}-\mathrm{a}^{2}}}{\mathrm{c}}\right.\right. \\
& \left.\times \tan \left(\frac{\sqrt{\mathrm{c}^{2}-\mathrm{a}^{2}}}{2}(\xi+C)\right)\right]^{-1} \\
& \left.-12(\mathrm{a}-\mathrm{c})^{2}\left[1-\frac{\mathrm{a}}{\mathrm{c}}+\frac{\sqrt{\mathrm{c}^{2}-\mathrm{a}^{2}}}{\mathrm{c}} \tan \left(\frac{\sqrt{\mathrm{c}^{2}-\mathrm{a}^{2}}}{2}(\xi+\mathrm{C})\right)\right]^{-2}\right\} .
\end{aligned}
$$

By using of the (102) and Family 2, 3 and $\mathbf{5}$ respectively get to

$$
\begin{aligned}
\mathrm{u}_{14}(\xi)= & \frac{\left(\mathrm{m}^{2}+1\right)}{\mathrm{m}\left(\mathrm{a}^{2}+\mathrm{b}^{2}-\mathrm{c}^{2}\right)}\left\{-6(\mathrm{a}-\mathrm{c})(\mathrm{b}-\mathrm{c})+12(\mathrm{a}-\mathrm{c})(\mathrm{a}+\mathrm{b}-\mathrm{c})\left[1+\frac{\mathrm{a}}{\mathrm{b}-\mathrm{c}}\right.\right. \\
& \left.+\frac{\sqrt{\mathrm{a}^{2}+\mathrm{b}^{2}-\mathrm{c}^{2}}}{\mathrm{~b}-\mathrm{c}} \tanh \left(\frac{\sqrt{\mathrm{a}^{2}+\mathrm{b}^{2}-\mathrm{c}^{2}}}{2}(\xi+C)\right)\right]^{-1} \\
& -12(\mathrm{a}-\mathrm{c})^{2}\left[1+\frac{\mathrm{a}}{\mathrm{b}-\mathrm{c}}+\frac{\sqrt{\mathrm{a}^{2}+\mathrm{b}^{2}-\mathrm{c}^{2}}}{\mathrm{~b}-\mathrm{c}}\right. \\
& \left.\left.\times \tanh \left(\frac{\sqrt{\mathrm{a}^{2}+\mathrm{b}^{2}-\mathrm{c}^{2}}}{2}(\xi+C)\right)\right]^{-2}\right\}, \\
\mathrm{u}_{15}(\xi)= & \frac{\left(\mathrm{m}^{2}+1\right)}{\mathrm{m}\left(\mathrm{a}^{2}+\mathrm{b}^{2}\right)}\left\{-6 \mathrm{ab}+12 \mathrm{a}(\mathrm{a}+\mathrm{b})\left[1+\frac{\mathrm{a}}{\mathrm{b}}+\frac{\sqrt{\mathrm{a}^{2}+\mathrm{b}^{2}}}{\mathrm{~b}} \tanh \left(\frac{\sqrt{\mathrm{a}^{2}+\mathrm{b}^{2}}}{2}(\xi+C)\right)\right]^{-1}\right. \\
& \left.+3 \mathrm{a}^{2}\left[1+\frac{\mathrm{a}}{\mathrm{b}}+\frac{\sqrt{\mathrm{a}^{2}+\mathrm{b}^{2}}}{\mathrm{~b}} \tanh \left(\frac{\sqrt{\mathrm{a}^{2}+\mathrm{b}^{2}}}{2}(\xi+C)\right)\right]^{-2}\right\},
\end{aligned}
$$




$$
\begin{aligned}
\mathrm{u}_{16}(\xi)= & \frac{\left(\mathrm{m}^{2}+1\right)}{\mathrm{m}\left(\mathrm{b}^{2}-\mathrm{c}^{2}\right)}\left\{6 \mathrm{c}(\mathrm{b}-\mathrm{c})-12 \mathrm{c}(\mathrm{b}-\mathrm{c})\left[1+\frac{\sqrt{\mathrm{b}^{2}-\mathrm{c}^{2}}}{\mathrm{~b}-\mathrm{c}} \tanh \left(\frac{\sqrt{\mathrm{b}^{2}-\mathrm{c}^{2}}}{2}(\xi+C)\right)\right]^{-1}\right. \\
& \left.-12 \mathrm{c}^{2}\left[1+\frac{\sqrt{\mathrm{b}^{2}-\mathrm{c}^{2}}}{\mathrm{~b}-\mathrm{c}} \tanh \left(\frac{\sqrt{\mathrm{b}^{2}-\mathrm{c}^{2}}}{2}(\xi+C)\right)\right]^{-2}\right\}
\end{aligned}
$$

By using of the (102) and Family 13 and $\mathbf{1 4}$ respectively can be written as

$$
\begin{aligned}
\mathrm{u}_{17}(\xi)= & \frac{\left(\mathrm{m}^{2}+1\right)}{\mathrm{m}}\left\{-\frac{12 \mathrm{a}(\mathrm{a}+\mathrm{b})}{\mathrm{b}^{2}}+\frac{24 \mathrm{a}(\mathrm{b}+2 \mathrm{a})}{\mathrm{b}^{2}}\left[1+\frac{\mathrm{e}^{\mathrm{b}(\xi+\mathrm{C})}+\mathrm{b}-\mathrm{a}}{\mathrm{e}^{\mathrm{b}(\xi+\mathrm{C})}-\mathrm{b}-\mathrm{a}}\right]^{-1}\right. \\
& \left.-\frac{48 \mathrm{a}^{2}}{\mathrm{~b}^{2}}\left[1+\frac{\mathrm{e}^{\mathrm{b}(\xi+\mathrm{C})}+\mathrm{b}-\mathrm{a}}{\mathrm{e}^{\mathrm{b}(\xi+\mathrm{C})}-\mathrm{b}-\mathrm{a}}\right]^{-2}\right\}, \\
\mathrm{u}_{18}(\xi)= & \frac{\left(\mathrm{m}^{2}+1\right)}{\mathrm{m}}\left\{\frac{12 \mathrm{c}(\mathrm{a}-\mathrm{c})}{\mathrm{a}^{2}}+\frac{12(\mathrm{a}-\mathrm{c})(\mathrm{a}-2 \mathrm{c})}{\mathrm{a}^{2}}\left[1-\frac{\mathrm{ae}}{\mathrm{ce}^{\mathrm{a}(\xi+\mathrm{C})}-1}\right]^{-1}\right. \\
& \left.-\frac{12(\mathrm{a}-\mathrm{c})^{2}}{\mathrm{a}^{2}}\left[1-\frac{\mathrm{ae} \mathrm{e}^{\mathrm{a}(\xi+\mathrm{C})}}{\mathrm{ce} \mathrm{e}^{\mathrm{a}(\xi+\mathrm{C})}-1}\right]^{2}\right\},
\end{aligned}
$$

where $\xi=\frac{1}{m} \sqrt{\frac{-\left(m^{2}+1\right)}{a^{2}+b^{2}-c^{2}}}(x-m t)$.

Set III:

$$
\begin{gathered}
\mathrm{m}=\mathrm{m}, \mu= \pm \frac{1}{\mathrm{~m}} \sqrt{\frac{\mathrm{m}^{2}+1}{\mathrm{a}^{2}+\mathrm{b}^{2}-\mathrm{c}^{2}}}, \mathrm{~A}_{0}=\frac{2\left(\mathrm{~m}^{2}+1\right)\left(\mathrm{a}^{2}+3 a b-3 a c+\mathrm{b}^{2}+2 \mathrm{c}^{2}-3 \mathrm{bc}\right)}{\mathrm{m}\left(\mathrm{a}^{2}+\mathrm{b}^{2}-\mathrm{c}^{2}\right)}, \\
\mathrm{B}_{1}=-\frac{12(\mathrm{a}-\mathrm{c})(\mathrm{a}+\mathrm{b}-\mathrm{c})\left(\mathrm{m}^{2}+1\right)}{\mathrm{m}\left(\mathrm{a}^{2}+\mathrm{b}^{2}-\mathrm{c}^{2}\right)}, \mathrm{B}_{2}=\frac{12(\mathrm{a}-\mathrm{c})^{2}\left(\mathrm{~m}^{2}+1\right)}{\mathrm{m}\left(\mathrm{a}^{2}+\mathrm{b}^{2}-\mathrm{c}^{2}\right)}, \mathrm{A}_{1}=0, \mathrm{~A}_{2}=0, \\
\mathrm{u}(\xi)=\mathrm{A}_{0}+\mathrm{B}_{1}\left[1+\tan \left(\frac{\Phi(\xi)}{2}\right)\right]^{-1}+\mathrm{B}_{2}\left[1+\tan \left(\frac{\Phi(\xi)}{2}\right)\right]^{-2},
\end{gathered}
$$

where a, b and c are arbitrary constants. By using of the (107) and Family $\mathbf{1 ,} \mathbf{4}$ respectively get to

$$
\begin{aligned}
\mathrm{u}_{19}(\xi)= & \frac{2\left(\mathrm{~m}^{2}+1\right)}{\mathrm{m}\left(\mathrm{a}^{2}+\mathrm{b}^{2}-\mathrm{c}^{2}\right)}\left\{\mathrm{a}^{2}+3 \mathrm{ab}-3 \mathrm{ac}+\mathrm{b}^{2}+2 \mathrm{c}^{2}-3 \mathrm{bc}-6(\mathrm{a}-\mathrm{c})(\mathrm{a}+\mathrm{b}-\mathrm{c})\right. \\
& \times\left[1+\frac{\mathrm{a}}{\mathrm{b}-\mathrm{c}}-\frac{\sqrt{\mathrm{c}^{2}-\mathrm{b}^{2}-\mathrm{a}^{2}}}{\mathrm{~b}-\mathrm{c}} \tan \left(\frac{\sqrt{\mathrm{c}^{2}-\mathrm{b}^{2}-\mathrm{a}^{2}}}{2}(\xi+C)\right)\right]^{-1}
\end{aligned}
$$




$$
\begin{aligned}
& \left.+6(a-c)^{2}\left[1+\frac{a}{b-c}-\frac{\sqrt{c^{2}-b^{2}-a^{2}}}{b-c} \tan \left(\frac{\sqrt{c^{2}-b^{2}-a^{2}}}{2}(\xi+C)\right)\right]^{-2}\right\}, \\
u_{20}(\xi)= & \frac{2\left(m^{2}+1\right)}{m\left(a^{2}-c^{2}\right)}\left\{a^{2}-3 a c+2 c^{2}-6(a-c)(a-c)\left[1-\frac{a}{c}+\frac{\sqrt{c^{2}-a^{2}}}{c}\right.\right. \\
& \left.\times \tan \left(\frac{\sqrt{c^{2}-a^{2}}}{2}(\xi+C)\right)\right]^{-1}+6(a-c)^{2}\left[1-\frac{a}{c}+\frac{\sqrt{c^{2}-a^{2}}}{c}\right. \\
& \left.\left.\times \tan \left(\frac{\sqrt{c^{2}-a^{2}}}{2}(\xi+C)\right)\right]^{-2}\right\}
\end{aligned}
$$

By using of the (107) and Family $\mathbf{2 , 3}$ and $\mathbf{5}$ respectively get to

$$
\begin{aligned}
& \mathrm{u}_{21}(\xi)=\frac{2\left(\mathrm{~m}^{2}+1\right)}{\mathrm{m}\left(\mathrm{a}^{2}+\mathrm{b}^{2}-\mathrm{c}^{2}\right)}\left\{\mathrm{a}^{2}+3 \mathrm{ab}-3 \mathrm{ac}+\mathrm{b}^{2}+2 \mathrm{c}^{2}-3 \mathrm{bc}-6(\mathrm{a}-\mathrm{c})(\mathrm{a}+\mathrm{b}-\mathrm{c})\right. \\
& \times\left[1+\frac{a}{b-c}+\frac{\sqrt{a^{2}+b^{2}-c^{2}}}{b-c} \tanh \left(\frac{\sqrt{a^{2}+b^{2}-c^{2}}}{2}(\xi+C)\right)\right]^{-1} \\
& +6(a-c)^{2}\left[1+\frac{a}{b-c}+\frac{\sqrt{a^{2}+b^{2}-c^{2}}}{b-c}\right. \\
& \left.\left.\times \tanh \left(\frac{\sqrt{a^{2}+b^{2}-c^{2}}}{2}(\xi+C)\right)\right]^{-2}\right\} \\
& \mathrm{u}_{22}(\xi)=\frac{2\left(\mathrm{~m}^{2}+1\right)}{\mathrm{m}\left(\mathrm{a}^{2}+\mathrm{b}^{2}\right)}\left\{\mathrm{a}^{2}+3 \mathrm{ab}+\mathrm{b}^{2}+-6 \mathrm{a}(\mathrm{a}+\mathrm{b})\left[1+\frac{\mathrm{a}}{\mathrm{b}}+\frac{\sqrt{\mathrm{a}^{2}+\mathrm{b}^{2}}}{\mathrm{~b}}\right.\right. \\
& \left.\times \tanh \left(\frac{\sqrt{\mathrm{a}^{2}+\mathrm{b}^{2}}}{2}(\xi+\mathrm{C})\right)\right]^{-1} \\
& \left.+6 a^{2}\left[1+\frac{a}{b}+\frac{\sqrt{a^{2}+b^{2}}}{b} \tanh \left(\frac{\sqrt{a^{2}+b^{2}}}{2}(\xi+C)\right)\right]^{-2}\right\}, \\
& \mathrm{u}_{23}(\xi)=\frac{2\left(\mathrm{~m}^{2}+1\right)}{\mathrm{m}\left(\mathrm{b}^{2}-\mathrm{c}^{2}\right)}\left\{\mathrm{b}^{2}+2 \mathrm{c}^{2}-3 \mathrm{bc}+6 \mathrm{c}(\mathrm{b}-\mathrm{c})\left[1+\frac{\sqrt{\mathrm{b}^{2}-\mathrm{c}^{2}}}{\mathrm{~b}-\mathrm{c}}\right.\right. \\
& \left.\times \tanh \left(\frac{\sqrt{\mathrm{b}^{2}-\mathrm{c}^{2}}}{2}(\xi+\mathrm{C})\right)\right]^{-1} \\
& \left.+6 c^{2}\left[1+\frac{\sqrt{b^{2}-c^{2}}}{b-c} \tanh \left(\frac{\sqrt{b^{2}-c^{2}}}{2}(\xi+C)\right)\right]^{-2}\right\} \text {. }
\end{aligned}
$$


By using of the (107) and Family 7 we get

$$
\begin{gathered}
\mathrm{u}_{24}(\xi)=\frac{2\left(\mathrm{~m}^{2}+1\right)}{\mathrm{m}}\left\{1-6\left[1+\tan \left(\frac{1}{2} \arctan \left[\frac{2 \mathrm{e}^{\mathrm{a}(\xi+\mathrm{C})}}{\mathrm{e}^{2 \mathrm{a}(\xi+\mathrm{C})}+1}, \frac{\mathrm{e}^{2 \mathrm{a}(\xi+\mathrm{C})}-1}{\mathrm{e}^{2 \mathrm{a}(\xi+\mathrm{C})}+1}\right]\right)\right]^{-1}\right. \\
\left.+6\left[1+\tan \left(\frac{1}{2} \arctan \left[\frac{2 \mathrm{e}^{\mathrm{a}(\xi+\mathrm{C})}}{\mathrm{e}^{2 \mathrm{a}(\xi+\mathrm{C})}+1}, \frac{\mathrm{e}^{2 \mathrm{a}(\xi+\mathrm{C})}-1}{\mathrm{e}^{2 \mathrm{a}(\xi+\mathrm{C})}+1}\right]\right)\right]^{-2}\right\} .
\end{gathered}
$$

By using of the (107) and Family 13 and $\mathbf{1 4}$ respectively can be written as

$$
\begin{aligned}
& \mathrm{u}_{25}(\xi)=\frac{2\left(\mathrm{~m}^{2}+1\right)}{\mathrm{m}}\left\{\frac{\mathrm{b}^{2}+6 \mathrm{ab}+6 \mathrm{a}^{2}}{\mathrm{~b}^{2}}-\frac{12 \mathrm{a}(\mathrm{b}+2 \mathrm{a})}{\mathrm{b}^{2}}\left[1+\frac{\mathrm{e}^{\mathrm{b}(\xi+\mathrm{C})}+\mathrm{b}-\mathrm{a}}{\mathrm{e}^{\mathrm{b}(\xi+C)}-\mathrm{b}-\mathrm{a}}\right]^{-1}\right. \\
& \left.+\frac{24 \mathrm{a}^{2}}{\mathrm{~b}^{2}}\left[1+\frac{\mathrm{e}^{\mathrm{b}(\xi+\mathrm{C})}+\mathrm{b}-\mathrm{a}}{\mathrm{e}^{\mathrm{b}(\xi+C)}-\mathrm{b}-\mathrm{a}}\right]^{-2}\right\} \\
& \mathrm{u}_{26}(\xi)=\frac{2\left(\mathrm{~m}^{2}+1\right)}{\mathrm{m}}\left\{\frac{\mathrm{a}^{2}-6 \mathrm{ac}+6 \mathrm{c}^{2}}{\mathrm{a}^{2}}-\frac{12(\mathrm{a}-\mathrm{c})(\mathrm{a}-2 \mathrm{c})}{\mathrm{a}^{2}}\left[1+\frac{\mathrm{ae}}{\mathrm{ce}^{\mathrm{a}(\xi+\mathrm{C})}-1}\right]^{-1}\right.
\end{aligned}
$$

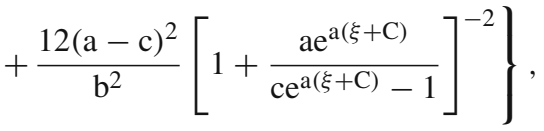

where $\xi=\frac{1}{m} \sqrt{\frac{m^{2}+1}{a^{2}+b^{2}-c^{2}}}(x-m t)$.

\section{Conclusion}

In the present work, we successfully obtained the exact solutions of the (2+1)-dimensional Zoomeron equation, the Duffing equation and the SRLW equation with the improved $\tan (\Phi(\xi) / 2)$-expansion method. By new scheme we established solitary solutions are include four type namely, triangular functions solutions, exponential solutions and rational solutions. The applied method will be used in further works to establish more entirely new solutions for the (2+1)-dimensional Zoomeron equation, the Duffing equation and the SRLW equation. This paper has shown the new method is sufficient incentive to seek more new exact soliton solutions of NEEs in mathematical physical. We found in this work the obtained results for the nonlinear aforementioned equations give very good results even very further of applied method in [5]. It can be concluded that this method is a very powerful and efficient technique in finding exact solutions for wide classes of problems. The performance of this method is reliable and effective and gives more solutions.

\section{References}

1. Magin, R.L.: Fractional calculus in bioengineering. Crit. Rev. Biomed. Eng. 32, 1-104 (2004)

2. Freed, A.D., Diethelm, K.: Fractional calculus in biomechanics: a 3D viscoelastic model using regularized fractional derivative kernels with application to the human calcaneal fat pad. Biomech. Model. Mech. 5, 203-215 (2006) 
3. Vosika, Z.B., Lazovic, G.M., Misevic, G.N., Simic-Krstic, J.B.: Fractional calculus model of electrical impedance applied to human skin. PLoS One 8, 1-12 (2013)

4. Hilfer, R.: Applications of Fractional Calculus in Physics. World Scientific Publishing Company, Singapore (2000)

5. Qawasmeh, A.: Soliton solutions of (2+1)-dimensional Zoomeron equation, the Duffing equation and the SRLW equation. J. Math. Comput. Sci. 3, 1475-1480 (2013)

6. Alquran, M., Al-Khaled, K.: Mathematical methods for a reliable treatment of the (2+1)-dimensional Zoomeron equation. Math. Sci. 6, 11 (2012)

7. Calogero, F., Degasperis, A.: Nonlinear evolution equations solvable by the inverse spectral transform I. Il Nuovo Cim. B 32, 201-242 (1976)

8. Abazari, R.: The solitary wave solutions of Zoomeron equation. Appl. Math. Sci. 59, 2943-2949 (2011)

9. Khan, K., Akbar, M.A.: Traveling wave solutions of the (2+1)-dimensional Zoomeron equation and the Burgers equations via the MSE method and the Exp-function method. Ain Shams Eng. J. 5, 247-256 (2014)

10. Bekir, A., Taşcan, F., Ünsal, Ö.: Exact solutions of the Zoomeron and Klein-Gordon-Zakharov equations. J. Assoc. Arab Univ. Basic Appl. Sci. 17, 1-5 (2014)

11. Balaji, S.: A new approach for solving Duffing equations involving both integral and non-integral forcing terms. Ain Shams Eng. J. 5, 985-990 (2014)

12. Manafian, J., Zamanpour, I.: Exact travelling wave solutions of the symmetric regularized long wave (SRLW) using analytical methods. Stat. Optim. Inf. Comput. 2, 47-55 (2014)

13. Xu, F.: Application of Exp-function method to symmetric regularized long wave (SRLW) equation. Phys. Comput. 372, 252-257 (2008)

14. Chand, F., Malik, A.K.: Exact traveling wave solutions of some nonlinear equations using (G'/G)expansion method. Int. J. Nonlinear Sci. 14, 416-424 (2012)

15. Hirota, R.: The Direct Method in Soliton Theory. Cambridge University Press, Cambridge (2004)

16. Dehghan, M., Manafian, J., Saadatmandi, A.: Solving nonlinear fractional partial differential equations using the homotopy analysis method. Numer. Methods Partial Differ. Equ. 26, 448-479 (2010)

17. He, J.H.: Variational iteration method a kind of non-linear analytical technique: some examples. Int. J. Nonlinear Mech. 34, 699-708 (1999)

18. Dehghan, M., Manafian, J.: The solution of the variable coefficients fourth-order parabolic partial differential equations by homotopy perturbation method. Z. Naturforsch 64a, 420-430 (2009)

19. Dehghan, M., Manafian, J., Saadatmandi, A.: Application of semi-analytic methods for the FitzhughNagumo equation, which models the transmission of nerve impulses. Math. Methods. Appl. Sci 33, 1384-1398 (2010)

20. Eslami, M., Mirzazadeh, M.: Exact solutions of modified Zakharov-Kuznetsov equation by the homogeneous balance method. Ain Shams Eng. J. 5(1), 221-225 (2014)

21. Wazwaz, A.M.: Travelling wave solutions for combined and double combined sine-cosine-Gordon equations by the variable separated ODE method. Appl. Math. Comput. 177, 755-760 (2006)

22. Manafian Heris, J., Lakestani, M.: Solitary wave and periodic wave solutions for variants of the KdVBurger and the $\mathrm{K}(\mathrm{n}, \mathrm{n})$-Burger equations by the generalized tanh-coth method. Commun. Num. Anal. 2013, 1-18 (2013)

23. Menga, X.H., Liua, W.J., Zhua, H.W., Zhang, C.Y., Tian, B.: Multi-soliton solutions and a Bäcklund transformation for a generalized variable-coefficient higher-order nonlinear Schrö dinger equation with symbolic computation. Phys. A. 387, 97-107 (2008)

24. Fazli Aghdaei, M., Manafianheris, J.: Exact solutions of the couple Boiti-Leon-Pempinelli system by the generalized $\left(\frac{\mathrm{G}^{\prime}}{\mathrm{G}}\right)$-expansion method. J. Math. Ext. 5, 91-104 (2011)

25. Mirzazadeh, M., Eslami, M., Biswas, A.: Soliton solutions of the generalized Klein-Gordon equation by using G'/G-expansion method. Comput. Appl. Math. 33(3), 831-839 (2014)

26. Mirzazadeh, M., Eslami, M., Milovic, D., Biswas, A.: Topological solitons of resonant nonlinear Schödinger'sequation with dual-power law nonlinearity by G'/G-expansion technique. Optik Int. J. Light Electron Opt. 125(19), 5480-5489 (2014)

27. Biswas, A., Mirzazadeh, M., Eslami, M.: Dispersive dark optical soliton with Schödinger-Hirota equation by G'/G-expansion approach in power law medium. Optik Int. J. Light Electron Opt. 125(16), 4215-4218 (2014)

28. Dehghan, M., Manafian Heris, J., Saadatmandi, A.: Application of the Exp-function method for solving a partial differential equation arising in biology and population genetics. Int. J. Numer. Methods Heat Fluid Flow 21, 736-753 (2011)

29. Dehghan, M., Manafian Heris, J., Saadatmandi, A.: Analytical treatment of some partial differential equations arising in mathematical physics by using the Exp-function method. Int. J. Mod. Phys. B 25, 2965-2981 (2011) 
30. Manafian Heris, J., Bagheri, M.: Exact solutions for the modified KdV and the generalized KdV equations via Exp-function method. J. Math. Ext. 4, 77-98 (2010)

31. Jawad, A.J.M., Petkovic, M.D., Biswas, A.: Modified simple equation method for nonlinear evolution equations. Appl. Math. Comput. 217, 869-877 (2010)

32. Eslami, M., Mirzazadeh, M., Biswas, A.: Soliton solutions of the resonant nonlinear Schrödinger's equation in optical fibers with time-dependent coefficients by simplest equation approach. J. Mod. Opt. 60(19), $1627-1636$ (2013)

33. Biswas, A., Mirzazadeh, M., Savescu, M., Milovic, D., Khan, K.R., Mahmood, M.F., Belic, M.: Singular solitons in optical metamaterials by ansatz method and simplest equation approach. J. Mod. Opt. 61(19), $1550-1555$ (2014)

34. Eslami, M., Vajargah, B.F., Mirzazadeh, M., Biswas, A.: Application of first integral method to fractional partial differential equations. Indian J. Phys. 88(2), 177-184 (2014)

35. Eslami, M., Mirzazadeh, M., Vajargah, B.F., Biswas, A.: Optical solitons for the resonant nonlinear Schrödinger's equation with time-dependent coefficients by the first integral method. Optik Int. J. Light Electron Opt. 125(13), 3107-3116 (2014)

36. Mirzazadeh, M., Eslami, M., Biswas, A.: Solitons and periodic solutions to a couple of fractional nonlinear evolution equations. Pramana 82(3), 465-476 (2014)

37. Eslami, M., Mirzazadeh, M.: Topological 1-soliton solution of nonlinear Schrödinger equation with dualpower law nonlinearity in nonlinear optical fibers. Eur. Phys. J. Plus 128(11), 1-7 (2013)

38. Nazarzadeh, A., Eslami, M., Mirzazadeh, M.: Exact solutions of some nonlinear partial differential equations using functional variable method. Pramana 81(2), 225-236 (2013)

39. Mirzazadeh, M., Eslami, M.: Exact solutions for nonlinear variants of Kadomtsev-Petviashvili (n, n) equation using functional variable method. Pramana 81(6), 911-924 (2013)

40. Mirzazadeh, M., Eslami, M., Vajargah, B.F., Biswas, A.: Optical solitons and optical rogons of generalized resonant dispersive nonlinear Schr ödinger's equation with power law nonlinearity. Optik Int. J. Light Electron Opt. 125(16), 4246-4256 (2014)

41. Malfliet, W.: Solitary wave solutions of nonlinear wave equations. Am. J. Phys. 60, 650-654 (1992)

42. Malfliet, W., Hereman, Willy: The tanh method: II. Perturbation technique for conservative systems. Phys. Scr. 54, 569-575 (1996)

43. Naher, H., Abdullah, F.A.: New approach of (G'/G)-expansion method and new approach of generalized $\left(G^{\prime} / G\right)$-expansion method for nonlinear evolution equation. AIP Adv. 3, 032116 (2013)

44. Manafian Heris, J., Lakestani, M.: Exact solutions for the integrable sixth-order Drinfeld-SokolovSatsuma-Hirota system by the analytical methods. Int. Sch. Res. Not. 2014, 840689 (2014)

45. Wang, M., Li, X., Zhang, J.: The $\left(\frac{G^{\prime}}{G}\right)$-expansion method and travelling wave solutions of nonlinear evolution equations in mathematical physics. Phys. Lett. A 372, 417-423 (2008)

46. Zhang, J., Wei, X., Lu, Y.: A generalized ( $\left.\frac{G^{\prime}}{G}\right)$-expansion method and its applications evolution equations in mathematical physics. Phys. Lett. A 372, 3653-3658 (2008)

47. Fan, E.: Extended tanh-function method and its applications to nonlinear equations. Phys. Lett. A 277, 212-218 (2000)

48. Wazwaz, A.M.: The tanh-coth method for new compactons and solitons solutions for the $K(n, n)$ and the $K(n+1, n+1)$ equations. Chaos Solitons Fractals 188, 1930-1940 (2007)

49. Wazwaz, A.M.: The extended tanh method for new solitons solutions for many forms of the fifth-order KdV equations. Appl. Math. Comput. 184, 1002-1014 (2007) 